DAMTP 91-34

LPTHE 91-61

NBI-HE 91-55

UGMS 91-23

\title{
Chern-Simons Theory as the Large Mass Limit of Topologically Massive Yang-Mills Theory
}

\author{
G. Giavarini \\ DAMTP, University of Cambridge \\ Silver Street, Cambridge CB3 9EW, UK \\ and \\ Laboratoire de Physique Théorique et Hautes Energies, Université Paris VI-VII * \\ Tour 14-24, 5 eme étage, 2 Place Jussieu, 75251 Paris Cedex 05, France \\ C. P. Martin \\ DAMTP, University of Cambridge \\ Silver Street, Cambridge CB3 9EW, UK \\ and \\ Department of Mathematics and Statistics, University of Guelph ${ }^{*}$ \\ Guelph, Ontario N1G 2W1, Canada \\ F. Ruiz Ruiz \\ The Niels Bohr Institute, University of Copenhagen \\ Blegdamsvej 17, DK-2100 Copenhagen Ø, Denmark
}

\footnotetext{
* Present address.
} 


\begin{abstract}
We study quantum Chern-Simons theory as the large mass limit of the limit $D \rightarrow 3$ of dimensionally regularized topologically massive YangMills theory. This approach can also be interpreted as a BRS-invariant hybrid regularization of Chern-Simons theory, consisting of a highercovariant derivative Yang-Mills term plus dimensional regularization. Working in the Landau gauge, we compute radiative corrections up to second order in perturbation theory and show that there is no two-loop correction to the one-loop shift $k \rightarrow k+c_{V}, k$ being the bare ChernSimons parameter. In passing we also prove by explicit computation that topologically massive Yang-Mills theory is UV finite.
\end{abstract}




\section{Introduction}

Some ten years ago it came to light that massive non-abelian gauge theories can be constructed in three dimensions without breaking local gauge invariance nor introducing in the classical lagrangian fields other than the gauge field[1,2]. A 3-dimensional alternative to the Higgs and Schwinger mechanisms was thus discovered. Massive gauge excitations were obtained by adding to the standard Yang-Mills action the intrinsically 3-dimensional Chern-Simons (CS) term, i.e. the integral over the spacetime manifold of the CS 3-form. The resulting gaugeinvariant theory was named topologically massive Yang-Mills (TMYM) theory[3]. The parameter, say $m$, in front of the CS term gives the bare mass of the gauge field, its square being the pole of the propagator in Minskowski spacetime, and is sometimes called topological mass. The CS term has some interesting properties. On the one hand, it is invariant under local gauge transformations connected to the identity and is a topological invariant. On the other, it is neither time-reversal invariant nor parity-preserving, and changes under topologically non-trivial gauge transformations. Invariance of the path integral under topologically non-trivial gauge transformations demands quantizing the ratio of the bare mass and the square of the coupling constant[4].

Some of the perturbative properties of TMYM theory have been studied in refs. [3,5]. In particular, UV finiteness for the theory has been shown at the oneloop level and anticipated to occur at two loops [3] if gauge invariance is respected by the UV divergent contributions from every two-loop diagram. This is however a non-trivial issue and its solution requires either $(i)$ a regularization-independent analysis, (ii) a gauge-invariant regularization, or (iii) an explicit computation. As an intermediate step towards the final goal of this paper, we will show by following the last two approaches that indeed no UV divergences arise at two loops and beyond. TMYM theory will thus turn out to be UV finite, although it is only superrenormalizable by power counting. In [5] the zero-momentum limit of 1PI Green functions with less than four external legs was proved to exist at every 
order in the number of loops. The topological CS term therefore improves the perturbative bad zero-momentum behaviour [1] of the corresponding massless 3dimensional Yang-Mills theory.

Two years ago a theory whose classical action is only the CS term was proposed [6]. The theory is called Chern-Simons theory and, being metric-independent, is a particular instance of the so-called topological field theories[7] (see[8] for a recent review). Topological field theories lack physical local degrees of freedom but have nonetheless applications to both Mathematics and Physics [8]. In particular, CS theory provides, on the physical side, a 3-dimensional setting for the understanding of 2-dimensional conformal field theories [6,9]. It is also used in condensed matter physics, e.g. in the study of heteroconjectures or 3-dimensional gases of non-relativistic electrons[10]. On the mathematical side, CS theory with gauge group $S U(N)$ gives a 3-dimensional, quantum field theoretical definition of the Jones polynomial[11] of knot theory and its generalization to the HOMFLY polynomial[12]. These knot and link invariants can be obtained from the vacuum expectations values of Wilson loops [6]. It is important to remind that Wilson loops are both gauge-invariant and metric-independent so that they are observables of the theory.

CS theory can be quantized non-perturbatively within the canonical formalism, where exact results can be obtained[13] (see [14] for another non-perturbative approach). This fact does not make the perturbative analysis to be an academic exercise. On the contrary, since many results in the canonical formulation rely on Feynman path integrals, which are not completely well-defined mathematical objects, a check using techniques from perturbative field theory is in order. Of outstanding importance is to see whether it is possible to recover in perturbation theory the find [6] that the exact result for the vacuum expectation value of a Wilson loop is a function of $q=\exp \left(\frac{2 \pi i}{k+N}\right)$, where the bare CS parameter $k(>0)$ has been shifted to $k+N$. More precisely, one would like to see how it occurs in the perturbative framework the fact[15] that the monodromy parameter of the theory and the central extension of the corresponding 2-dimensional current algebra are 
$k+N$ and $k$ respectively, $k$ being the bare CS parameter. Furthermore, perturbative quantization of CS theory has led to analytic expressions for the coefficients of the Jones polynomial and its generalizations in terms of integrals involving the gauge propagator[16]. These coefficients are knot and link invariants and their meaning and properties remain largely unkown[17]. Having explicit analytic expressions for them may help to unravel their significance. This program has been started in [16], where new relationships for the HOMFLY coefficients have been obtained.

There are already a good many papers on perturbativel 6,58thaspley Most of them deal with the study of the Landau-gauge effective action and its properties. Although perturbative CS theory is not UV finite by power counting but merely renormalizable, both the beta function $[27,28]$ and the anomalous dimensions of the elementary fields [28] have been proved to vanish at any order in perturbation theory, in accordance with non-perturbative results (see also [24,33]). Several groups have undertaken the computation of the one-loop effective action by using a number of regularization methods [18-23]. The picture that has emerged from these calculations is that every BRS-invariant regularization method [6,1921] gives for the gauge-invariant part of the one-loop effective action the CS term with coefficient $k+N, k(>0)$ being the bare or classical CS parameter. Thus, the bare parameter $k$ is shifted to $k+N$ for all BRS-invariant regulators used as yet. On the other hand, this shift fails to occur for regularization methods that are not gauge-invariant $[18,24]$. One-loop results suggest therefore that BRSinvariant regulators will naturally provide a monodromy parameter $k+N(k>0)$, as non-perturbtive results demand. (We refer the reader to the conclusions for a further discussion of this idea). Of course, for a complete agreement with the hamiltonian formalism, there must be no corrections to $k+N$ beyond first order in perturbation theory [34].

The main purpose of this paper is to show the absence of two-loop corrections to the one-loop shift $k \rightarrow k+N$ for the BRS-invariant regularization first introduced in [21]. This regulator is a hybrid method obtained by adding to the CS action a 
Yang-Mills term and then, by regularizing the UV divergences that are left with a BRS-invariant dimensional regularization. In this way the regularized theory involves two regulating parameters, one is the dimensionality $D$ of spacetime (we will also use $\varepsilon=D-3$ ), and the other, which we call $m$, is a cut-off introduced by the Yang-Mills term. We shall define CS theory as the limit $D \rightarrow 3, m \rightarrow \infty$ of the regularized theory. After appropriate rescalings of the fields and coupling constant, the completely regularized CS theory will turn out to be a dimensionally regularized TMYM theory whose bare topological mass is $m$, the former YangMills regulating parameter. Thus CS theory can be viewed as the large mass limit of TMYM theory, provided the limit $D \rightarrow 3$ of dimensionally regularized TMYM theory exits. We shall show that the latter limit does exist (see Sects. 2 and 7 for a more precise discussion).

The layout of this paper is as follows. In Sect. 2 we introduce our explicitly BRS-invariant regularization method for CS theory and show how it can be interpreted as a dimensional regularization of TMYM theory. Sect. 3 is devoted to some theorems that simplify enormously the computation of the large mass limit of the integrals arising from the Feynman diagrams we will have to consider. The calculation of the 1PI Green functions needed to construct the local part of the effective action up to two loops is presented in Sects. 4, 5 and 6. For the sake of the reader, we collect in Sect. 7 the main steps leading to our proving the UV finiteness for TMYM theory. In Sect. 8 the complete local part of the bare effective action is obtained up to second order in perturbation theory by using BRS techniques and the values of the 1PI functions obtained in previous sections. The absence of two-loop corrections to the one-loop shift $k \rightarrow k+N$ is discussed in Sect. 9. Finally, Sect. 10 contains the conclusions we have drawn from the analyis reported here. We also include an appendix with the relevant integrals we have had to evaluate in our computations. 


\section{The large $m$ limit, Feynman rules and regularization}

Let us consider $\mathbb{R}^{3}$ with the euclidean metric and denote by $\mathcal{A}_{\mu}=\mathcal{A}_{\mu}^{a} T^{a}$ the $S U(N)$ gauge connection in the fundamental representation, $T^{a}$ being the antihermitean generators of the Lie algebra in this representation. We take the structure constants $f^{a b c}$ of $S U(N)$ to be completely antisymmetric in their indices, with $\left[T^{a}, T^{b}\right]=f^{a b c} T^{c}$. For the generators $T^{a}$ we choose the normalization $\operatorname{Tr}\left(T^{a} T^{b}\right)=\frac{1}{2} \delta^{a b}$. The corresponding BRS-invariant action in the Landau gauge for TMYM theory with bare mass $m>0$ and bare coupling constant $g_{t}$ is then given by $[2,3,5]$

$$
\begin{aligned}
S_{m}= & -i m \int d^{3} x \epsilon^{\mu \rho \nu}\left(\frac{1}{2} \mathcal{A}_{\mu}^{a} \partial_{\rho} \mathcal{A}_{\nu}^{a}+\frac{1}{3 !} g_{t} f^{a b c} \mathcal{A}_{\mu}^{a} \mathcal{A}_{\rho}^{b} \mathcal{A}_{\nu}^{c}\right) \\
& +\frac{1}{4} \int d^{3} x \mathcal{F}_{\mu \nu}^{a} \mathcal{F}_{\mu \nu}^{a}-\int d^{3} x\left(\mathcal{B}^{a} \partial \mathcal{A}^{a}-\bar{c}^{a} \partial \mathcal{D}^{a b} c^{b}\right),
\end{aligned}
$$

where $\mathcal{F}_{\mu \nu}^{a}=\partial_{\mu} \mathcal{A}_{\nu}^{a}-\partial_{\nu} \mathcal{A}_{\mu}^{a}+g_{t} f^{a b c} \mathcal{A}_{\mu}^{b} \mathcal{A}_{\nu}^{c}$ is the field strength and $\mathcal{D}_{\mu}^{a b}=$ $\partial_{\mu} \delta^{a b}+g_{t} f^{a c b} \mathcal{A}_{\mu}^{c}$ the covariant derivative. The mass dimensions of the fields $\mathcal{A}_{\mu}^{a}, \mathcal{B}^{a}, \bar{c}^{a}, c^{a}$ and of the coupling constant $g_{t}$ are respectively $1 / 2,3 / 2,0,1$ and $1 / 2$. As for $m$, it has dimensions of mass.

In turn, the BRS-invariant action in the Landau gauge for CS theory is defined to be

$$
\begin{aligned}
S= & -i \int d^{3} x \epsilon^{\mu \rho \nu}\left(\frac{1}{2} A_{\mu}^{a} \partial_{\rho} A_{\nu}^{a}+\frac{1}{3 !} g f^{a b c} A_{\mu}^{a} A_{\rho}^{b} A_{\nu}^{c}\right) \\
& -\int d^{3} x\left(b^{a} \partial A^{a}-\bar{c}^{a} \partial D^{a b} c^{b}\right),
\end{aligned}
$$

with $A_{\mu}^{a}$ is the gauge field on $\mathbb{R}^{3}$ and $D_{\mu}^{a b}=\partial_{\mu} \delta^{a b}+g f^{a c b} A_{\mu}^{c}$. The fields $A_{\mu}^{a}, b^{a}, \bar{c}^{a}$ and $c^{a}$ now have mass dimensions 1, 1, 0 and 1, respectively; the coupling constant $g$ is dimensionless.

It has been suggested[36] that the limit $m \rightarrow \infty$ of TMYM theory with classical action (2.1) should yield pure CS theory with classical action (2.2). The purpose of this section is to propose a way to define this limit so that it gives the desired result, at least in the highly non-trivial instances we have checked. 
We begin by considering tree-level Green functions for the field $\mathcal{A}_{\mu}^{a}$. By taking into acount the Feynman rules in ref. [5] one immediately realizes that their large $m$ limit vanishes. This is due to the fast oscillations as $m \rightarrow \infty$ of the Boltzman factor in the path integral, owing to the CS term in $S_{m}$. Thus, to obtain pure CS theory as the large $m$ limit of TMYM theory, the limit $m \rightarrow \infty$ cannot be directly taken over Green functions in terms of $g_{t}$ but some other way must be contrived. Notice that the dimensions of the fields and coupling constant in $S_{m}$ in eq. (2.1) do not match those of the corresponding fields and coupling constant in $S$ in eq. (2.2), being then little wonder that pure CS theory is not obtained by taking the large $m$ limit as above. To remedy this dimensional mismatch one introduces the following scalings of the fields $\mathcal{A}_{\mu}^{a}$ and $\mathcal{B}^{a}$ and the coupling constant $g_{t}$ :

$$
A_{\mu}^{a}=m^{1 / 2} \mathcal{A}_{\mu}^{a}, \quad b^{a}=m^{-1 / 2} \mathcal{B}^{a}, \quad g=m^{-1 / 2} g_{t} .
$$

We have used the same notation for the rescaled fields and coupling constant as in eq. (2.2) for reasons that will become clear in a moment. Notice that $g A=g_{t} \mathcal{A}$ and that the mass dimensions of $A_{\mu}^{a}, b^{a}$ and $g$ in eq. (2.3) are the same as those of the corresponding fields and coupling constant in the CS action (2.2). Similar scalings have been introduced by other authors in the context of CS quantum mechanics[37].

In terms of the new fields and coupling constant, the TMYM action (2.1) can be recast in the form

$$
\begin{aligned}
S_{m}= & -i \int d^{3} x \epsilon^{\mu \rho \nu}\left(\frac{1}{2} A_{\mu}^{a} \partial_{\rho} A_{\nu}^{a}+\frac{1}{3 !} g f^{a b c} A_{\mu}^{a} A_{\rho}^{b} A_{\nu}^{c}\right) \\
& +\frac{1}{4 m} \int d^{3} x F_{\mu \nu}^{a} F^{a \mu \nu}-\int d^{3} x s\left(\bar{c}^{a} \partial A^{a}+J^{a} A^{a}-H^{a} c^{a}\right),
\end{aligned}
$$

with $F_{\mu \nu}^{a}=\partial_{\mu} A_{\nu}^{a}-\partial_{\nu} A_{\mu}^{a}+g f^{a b c} A_{\mu}^{b} A_{\nu}^{c}$. With the purpose of computing the effective action using BRS techniques we have introduced external fields $J_{\mu}^{a}$ and $H^{a}$ 
coupled to the non-linear BRS transforms $s A_{\mu}^{a}$ and $s c^{a}$ respectively. The action on the fields of the BRS operator $s$ is:

$s A_{\mu}^{a}=\left(D_{\mu} c\right)^{a}, s \bar{c}^{a}=b^{a}, s c^{a}=-\frac{1}{2} g f^{a b c} c^{b} c^{c}, s b^{a}=0, s J_{\mu}^{a}=s H^{a}=0$

$D_{\mu}^{a b}$ being the covariant derivative in terms of $A_{\mu}^{a}$ and $g$.

The action $S_{m}$ is invariant under $s$, since $s$ itself is nilpotent, i.e. $s^{2}=0$, and the terms in $S_{m}$ that depend only on the gauge field are invariant under infinitesimal gauge transformations for any value of $g$. This brings along a comment on the quantization of $g$ as defined in eq. (2.3). It is well-known [3] that invariance of TMYM theory under gauge transformations with non-zero winding number demands $g_{t}$ in (2.1) to be quantized according to $4 \pi m / g_{t}^{2}=1,2,3, \ldots$ Consequently, $g$ in (2.3) must satisfy

$$
\frac{4 \pi}{g^{2}} \equiv k=1,2,3, \ldots
$$

These quantization conditions, however, require large values of the fields to occur and therefore they do not appear in the perturbative regime we shall consider in this paper.

The Feynman rules obtained from the action $S_{m}$ in eq. (2.4) are the following:

a) Propagators. The gauge field propagator is

$$
<A_{\mu}^{a}(p) A_{\nu}^{b}(-p)>=D_{\mu \nu}^{a b}(p)=\frac{\delta^{a b} m}{p^{2}\left(p^{2}+m^{2}\right)}\left(m \epsilon_{\mu \rho \nu} p^{\rho}+p^{2} g_{\mu \nu}-p_{\mu} p_{\nu}\right)
$$

For the ghost and auxiliary field we have:

$$
\begin{gathered}
<c^{a}(p) \bar{c}^{b}(p)>=D^{a b}(p)=-\frac{\delta^{a b}}{p^{2}} \\
<b^{a}(p) A_{\mu}^{b}(-p)>=D_{\mu}^{a b}(p)=i \delta^{a b} \frac{p_{\mu}}{p^{2}} .
\end{gathered}
$$


b) Vertices. The three- and four-gauge vertices are

$$
\begin{gathered}
V_{\mu \nu \rho}^{a b c}(p, q, r)=i g f^{a b c}\left\{\epsilon_{\mu \nu \rho}+\frac{1}{m}\left[(q-r)_{\mu} g_{\nu \rho}+(r-p)_{\nu} g_{\rho \mu}+(p-q)_{\rho} g_{\mu \nu}\right]\right\} \\
V_{\mu \nu \rho \sigma}^{a b c d}=-\frac{g^{2}}{m}\left[f^{a b e} f^{c d e}\left(g_{\mu \rho} g_{\nu \sigma}-g_{\nu \rho} g_{\mu \sigma}\right)+f^{c b e} f^{a d e}\left(g_{\mu \rho} g_{\nu \sigma}-g_{\nu \mu} g_{\rho \sigma}\right)\right. \\
\left.+f^{d b e} f^{c a e}\left(g_{\sigma \rho} g_{\nu \mu}-g_{\nu \rho} g_{\mu \sigma}\right)\right],
\end{gathered}
$$

while the ghost vertex reads

$$
V_{\mu}^{a b c}(q)=-i g f^{a b c} q_{\mu} .
$$

Finally, the gauge-ghost-external field vertex $J_{\mu}^{a} A_{\nu}^{b} c^{c}$ and the ghost-ghost-external field vertex $H^{a} c^{b} c^{c}$ are

$$
-g f^{a b c} g_{\mu \nu} \text { and } g f^{a b c}
$$

respectively. The diagramatic representation of these rules is depicted in Fig. 1.

Taking the large $m$ limit at fixed $g$ of (2.6)-(2.10) one recovers the Feynman rules for pure CS theory with coupling constant $g$, as obtained directly from the action (2.2). Hence, the definition of pure $S U(N)$ Chern-Simons theory as the large $m$ limit of $S U(N)$ topologically massive Yang-Mills theory arises naturally. In particular, we define the $E$-point gauge field Green function $G_{\mu_{1} \cdots \mu_{E}}^{a_{1} \cdots a_{E}}\left(p_{1}, \ldots, p_{E} ; g\right)$ for pure CS theory as the limit

$$
\begin{aligned}
G_{\mu_{1} \cdots \mu_{E}}^{a_{1} \cdots a_{E}}\left(p_{1}, \ldots, p_{E} ; g\right) & =\lim _{m \rightarrow \infty}<A_{\mu_{1}}^{a}\left(p_{1}\right) \cdots A_{\mu_{E}}^{a_{E}}\left(p_{E}\right)>(g) \\
& =\lim _{m \rightarrow \infty} m^{E / 2}<\mathcal{A}_{\mu_{1}}^{a}\left(p_{1}\right) \cdots \mathcal{A}_{\mu_{E}}^{a_{E}}\left(p_{E}\right)>(g) .
\end{aligned}
$$

Here we have explicitly written the dependence on $g$ to remind that the Green functions are evaluated at fixed (but arbitrary) $g$, not to be confused with $g_{t}$. 
In what follows we will omit this fact in the notation. The external momenta $p_{1}, \ldots, p_{E}$ are also arbitrary but lie in a bounded domain and are non-exceptional. Similar definitions can be introduced for Green functions involving auxiliary and/or ghost fields. All these Green functions are defined as formal series in powers of $g$ whose coefficients are obtained using Feynman diagramatic techniques.

Let us emphasize that, as already shown, eq. (2.12) holds at the tree-level and that our approach is to regard it as a definition for CS Green functions to all orders in perturbation theory. This paper is devoted to study the existence of the large $m$ limit up to two loops. In particular, following eq. (2.12) we will explicitly construct the local two-loop effective action for pure CS theory.

It should be noticed that the definition in eq. (2.12) is not yet meaningful in perturbation theory since TMYM theory is not UV finite by power counting. The superficial UV degree $\omega_{m}$ of a 1PI Feynman diagram with $E_{g}$ external gauge lines, $E_{g h}$ external ghost lines, $V_{3}$ vertices with three gauge legs, $V_{4}$ vertices with four gauge legs, $V_{g h}$ vertices of type $\bar{c} A c, V_{J}$ vertices of type $J A c$ and $V_{H}$ vertices of type $H c c$ is

$$
\omega_{m}=3-\frac{1}{2}\left(E_{g}+2 E_{g h}+V_{3}+V_{g h}+2 V_{4}+3 V_{J}+2 V_{H}\right) .
$$

We thus see that only a finite number of 1 PI diagrams have $\omega_{m} \geq 0$ so that TMYM theory is superrenormalizable. Let us spell out the 1PI functions that are primitively UV divergent. We shall not consider diagrams contributing to $\left\langle A_{\mu}^{a}\right\rangle$, since as we will show in Sect. 4, they vanish upon regularization owing to colour algebra. Then, the only superficially UV divergent Green functions at one loop are the gauge field two- and three-point functions and the ghost propagator, for which $\omega_{m}=1, \omega_{m}=0$ and $\omega_{m}=0$ respectively. At second order in perturbation theory, only 1PI diagrams contributing to the gauge field propagator have non-negative $\omega_{m}$, namely $\omega_{m}=0$. Regarding higher loop 1PI diagrams, they are all superficially UV convergent. To provide the superficially UV divergent TMYM 1PI diagrams with a well-defined mathematical meaning we will use dimensional regularization. 
Its implementation in the case at hand is not straightforward due to the presence of the Levi-Civita symbol and will be the subject of the next subsection. In this paper we will show by explicit computation that, once the UV divergences have been dimensionally regularized, the sum of all 1PI Feynman diagrams at a given order in perturbation theory is finite as $\varepsilon \rightarrow 0, \varepsilon=D-3$ being the dimensional regulator. This implies that there is no need of UV divergent counterterms to define the effective action for TMYM theory and that TMYM Green functions [entering in e.g. the RHS of eq. (2.12)] can be understood as the limit $D \rightarrow 3$ of the corresponding dimensionally regularized functions.

Let us point out that the action of eq. (2.4) can be thought of as generating a higher-covariant derivative regularization [38] of pure CS theory. As usual, such regularization is not enough as to regularize all 1PI diagrams and, in addition, a second regularization must be implemented. For computational purposes one may consider dimensional regularization. Thus, our proposal to obtain pure CS theory as the large $m$ limit of dimensioanlly regularized TMYM theory can be alternatively viewed as a hybrid regularization method: higher-covariant derivatives plus dimensional regularization. This method is characterized by two regulators: $\varepsilon$ and $m$. The prescription, in accordance with eq. (2.12), is to first send $\varepsilon$ to zero and then $m$ to infinity. Although there is no systematic study of such hybrid regularization methods, it may be argued that this double limit should yield a finite result, since the beta function $[27,28]$ and the anomalous dimensions of the elementary fields [28] vanish for CS theory in the Landau gauge. Our definition of the large $m$ limit would then make sense at any order in perturbation theory. We will see that our two-loop explicit computations are in agreement with this general statement, so in some sense we will have checked a particular case of hybrid regularization in a non-trivial context. Notice that the vanishing of the anomalous dimensions of the fields in both CS and TMYM theories implies that that the naïve dimensional matchings in eq. (2.3) are not destroyed by radiative corrections, at least in the Landau gauge.

Since TMYM theory involves the Levi-Civita tensor, the first difficulty one 
encounters when dimensionally regularizing the theory is to give a definition of the $n$-dimensional counterpart of $\epsilon_{\mu \rho \nu}$. In this paper we shall adopt the method proposed by 't Hooft and Veltman[39] and systematized by Breitenlohner and Maison[40] to incorporate parity violating objects such as $\epsilon_{\mu \rho \nu}$ within the framework of dimensional regularization. This method leads to the only known algebraically consistent definition of such objects, and has proved useful in perturbative computations in WZW[41] and two-dimensional non-linear sigma models[42]. It amounts to defining the $n$-dimensional $\epsilon_{\mu \rho \nu}$ as a completely antisymmetric object in its indices which satisfies the following identities:

$$
\begin{gathered}
\epsilon_{\mu_{1} \mu_{2} \mu_{3}} \epsilon_{\nu_{1} \nu_{2} \nu_{3}}=\sum_{\pi \epsilon P_{3}} \operatorname{sign}(\pi) \prod_{i=1}^{3} \tilde{g}_{\mu_{i} \nu_{\pi(i)}}, \\
\epsilon_{\mu_{1} \mu_{2} \mu_{3}} \hat{g}^{\mu_{3} \mu_{4}}=0 \quad, \quad \epsilon_{\mu_{1} \mu_{2} \mu_{3}} \hat{u}^{\mu_{3}}=0, \\
u_{\mu}=\tilde{u}_{\mu} \oplus \hat{u}_{\mu}, \quad g_{\mu \nu}=\tilde{g}_{\mu \nu} \oplus \hat{g}_{\mu \nu} \quad, \quad \tilde{g}_{\mu}^{\mu}=3 \quad, \quad \hat{g}_{\mu}^{\mu}=n-3,
\end{gathered}
$$

Here all indices run from 1 to $n, n$ an intenger larger or equal than $3, g_{\mu \nu}$ is the euclidean metric on $\mathbb{R}^{n}$, and $\tilde{g}_{\mu \nu}$ and $\hat{g}_{\mu \nu}$ are its projections onto the subspaces $\mathbb{R}^{3}$ and $\mathbb{R}^{n-3}$. For $n \geq 3$, twiddled and hatted objects should be understood in this way throughout this paper, i.e. as projections onto $\mathbb{R}^{3}$ and $\mathbb{R}^{n-3}$. Note that this definition of $\epsilon_{\mu \rho \nu}$ only preserves $S O(3) \otimes S O(n-3)$ covariance, rather than $S O(n)$. This makes computations more complicated but, on the other hand, guarantees algebraic consistency. It must be stressed that in dimensional regularization, when $n$ is promoted to a complex variable $D$, indices cease to have particular values and that $u_{\mu}, g_{\mu \nu}, \varepsilon_{\mu \nu \rho}, \ldots$ are defined through algebraic relations only [40], such as (2.14).

We can now proceed to dimensionally regularize TMYM theory. The simplest and most naïe approach is to promote the 3-dimensional Feynman rules of TMYM theory to $n$ dimensions and then use dimensional regularization techniques to evaluate the corresponding integrals. Despite the simplicity of this prescription, 
we will show that in the 3-dimensional limit the BRS identities hold. There is also the alternative and more sophisticated approach of constructing an explicitly BRS-invariant regularizing theory. In what follows we discuss both approaches and show that they provide the same result.

\subsection{Naïve dimensional regularization of TMYM theory}

The idea is to first replace the 3-dimensional Feynman rules (2.6)-(2.11) with a set of rules in $n$ dimensions. This is achieved by substituting the Levi-Civita symbol with the $n$-dimensional $\epsilon_{\mu \rho \nu}$ defined in eq. (2.14) and by considering the 3 vectors and the 3 -dimensional euclidean metric entering in (2.6)-(2.11) as $n$-vectors and the $n$-dimensional euclidean metric on $\mathbb{R}^{n}$. Next, one regards every TMYM diagram as given by these $n$-dimensional Feynman rules, with the proviso that integrals are formally defined in $n$ dimensions. Finally, one uses the techniques in [40] or [43] to continue Feynman integrals to complex values $D$ of $n$. In this way every diagram is expressed in terms of well-defined integrals for $D$ in some domain of the complex plane.

The main virtue of this approach is to keep $n$-dimensional propagators as simple as possible so that the algebra is manageable and, furthermore, the resulting integrals can be computed using elementary textbook techniques. All this is very desirable since we will carry out a computation already cumbersome by itself. Unfortunately, the dimensionally regularized Green functions obtained in this way do not satisfy the BRS identities. However, although BRS invariance is lost at the regularized level, the contributions that break the BRS identities vanish as $D \rightarrow 3$. Let us make more clear all these statements.

We begin by obtaining the expression of the breaking of the BRS identities in terms of Green functions. It is not difficult to see that the Feynman rules (2.6)(2.11) in $n$ dimensions, with $\varepsilon_{\mu \nu \rho}$ as in (2.14), come from the following action:

$$
S[J, H]=S_{m}^{n}[J, H]+S_{B},
$$


where $S_{m}^{n}[J, H]$ is local and given by

$$
\begin{aligned}
S_{m}^{n}[J, H]= & -i \int d^{n} x \epsilon^{\mu \rho \nu}\left(\frac{1}{2} A_{\mu}^{a} \partial_{\rho} A_{\nu}^{a}+\frac{1}{3 !} g f^{a b c} A_{\mu}^{a} A_{\rho}^{b} A_{\nu}^{c}\right) \\
& +\frac{1}{4 m} \int d^{n} x F_{\mu \nu}^{a} F^{a \mu \nu}-\int d^{n} x s\left(\bar{c}^{a} \partial A^{a}+J^{a} A^{a}-H^{a} c^{a}\right)
\end{aligned}
$$

and $S_{B}$ stands for the non-local contribution

$$
S_{B}=\frac{1}{2} \int d^{n} x d^{n} y A_{\mu}^{a}(x) O_{\mu \nu}(x-y) A_{\nu}^{a}(y) .
$$

The operator $O_{\mu \nu}(x-y)$ is given by

$$
O_{\mu \nu}(x-y)=\int \frac{d^{n} p}{(2 \pi)^{n}} O_{\mu \nu}(p) e^{i p(x-y)},
$$

with

$$
\begin{aligned}
O_{\mu \nu}(p)=\frac{m}{\left(p^{2}\right)^{2}+m^{2} \tilde{p}^{2}} & {\left[\hat{p}^{2}\left(-m \epsilon_{\mu \rho \nu} p^{\rho}+p^{2} g_{\mu \nu}-p_{\mu} p_{\nu}\right)\right.} \\
& \left.+\left(p^{2}+m^{2}\right)\left(\frac{\hat{p}^{2}}{p^{2}} p_{\mu} p_{\nu}+\tilde{p}^{2} \hat{g}_{\mu \nu}+\hat{p}_{\mu} \hat{p}_{\nu}-p_{\mu} \hat{p}_{\nu}-\hat{p}_{\mu} p_{\nu}\right)\right]
\end{aligned}
$$

The BRS operator $s$ in (2.16) is the $n$-dimensional version of that in three dimensions: it is given by eqs. (2.5) but now all functions and vectors are defined on $\mathbb{R}^{n}$. In the remainder of this section the operator $s$ should be understood in this $n$-dimensional sense.

To find the BRS identities we need the formal, perturbative partition function $Z[j, w, \bar{w}, \lambda ; J, H]$ corresponding to the action $S[J, H]$ :

$$
\begin{aligned}
Z[j, w, \bar{w}, \lambda ; J, H] & =\frac{1}{Z[0]} \int[d A][d c][d \bar{c}][d b] e^{-S[J, H]+\int d^{n} x(j A+\lambda b+\bar{w} c+\bar{c} w)} \\
& =\sum_{E=0}^{\infty} \int d^{n_{1}} x \cdots d^{n_{E}} x G\left(x_{1}, \ldots, x_{E}\right) f\left(x_{1}\right) \cdots f\left(x_{E}\right)
\end{aligned}
$$

Here $f(x)$ denotes any of the sources $j_{\mu}^{a}, w^{a}, \bar{w}^{a}, \lambda^{a}$ and/or the external fields $J_{\mu}^{a}, H^{a}$, and the Green functions $G\left(x_{1}, \ldots, x_{E}\right)$ are obtained by adding all nonvacuum Feynman diagrams with appropriate elementary fields and insertions of 
type $s A_{\mu}^{a}$ and $s c^{a}$. It is immediate to see that the BRS variation of the local action $S_{m}^{n}[J, H]$ vanishes so that

$$
s S[J, K]=s S_{B}=g f^{a b c} \int d^{n} x d^{n} y A_{\mu}^{a}(x) O_{\mu \nu}(x-y) A_{\nu}^{b}(y) c^{c}(y)
$$

This, together with the fact that the partition function is invariant under trnasformations (2.5), leads to

$$
\Sigma Z[j, w, \bar{w}, \lambda ; J, H]=B
$$

where $\Sigma$ is the functional operator

$$
\Sigma=\int d^{n} x\left(-j_{\mu}^{a} \frac{\delta}{\delta J_{\mu}^{a}}+\bar{w}^{a} \frac{\delta}{\delta H^{a}}-w^{a} \frac{\delta}{\delta \lambda^{a}}\right)
$$

and $B$ has the form

$$
B=\frac{1}{Z[0]} \int[d A][d c][d \bar{c}][d b]\left(s S_{B}\right) e^{-S[J, H]+\int d^{n} x(j A+\lambda b+\bar{w} c+\bar{c} w)}
$$

Eqs. (2.19) and (2.21) are both to be understood as the perturbation series obtained from the corresponding formal $n$-dimensional Feynman diagrams. That the formal manipulations leading to eqs. (2.19) and (2.21) make sense for the dimensionally regularized Green functions in (2.18) follows from properties of dimensionally regularized Feynman integrals [43]. Thus, eqs. (2.19) and (2.21), when interpreted in terms of Feynman diagrams, hold in dimensional regularization, where $n$ is promoted to a complex parameter $D$.

Once the breaking $B$ of the BRS identities has been obtained, the next step is to show that it goes to zero as $D$ approaches 3 . In doing so one has to prove 
that the limit $D \rightarrow 3$ of the Green function

$$
\begin{aligned}
g f^{a b c}\left\langle\int d^{D} x d^{D} y A_{\mu}^{a}(x) O_{\mu \nu}(x-y) A_{\nu}^{b}(y) c^{c}(y)\right. \\
\left.\left.\qquad \prod_{i=1}^{n_{1}} A_{\mu_{i}}^{a_{i}}\left(x_{i}\right) \prod_{i=1}^{n_{2}} b^{a_{i}}\left(x_{i}\right) \prod_{i=1}^{n_{3}} c^{a_{i}}\left(x_{i}\right) \prod_{i=1}^{n_{4}} \bar{c}^{a_{i}}\left(x_{i}\right) \prod_{i=1}^{n_{5}} s A_{\mu_{i}}^{a_{i}}\left(x_{i}\right) \prod_{i=1}^{n_{6}} s c^{a_{i}}\left(x_{i}\right)\right\}\right\rangle,
\end{aligned}
$$

vanishes for all values of $n_{1}, n_{2}, n_{3}, n_{4}, n_{5}$ and $n_{6}$ at any order in perturbation theory, the symbol $\langle\cdots\rangle$ denoting

$$
\frac{1}{Z[0]} \int[d A][d c][d \bar{c}][d b] \cdots e^{-S[0,0]} .
$$

Now, any dimensionally regularized contribution to the the Green function (2.22) can be arranged in the form

$$
\begin{aligned}
I_{\mu_{1} \cdots \mu_{A}}^{a_{1} \cdots a_{E}}\left(p_{1}, \cdots, p_{E}\right)=g f^{a b c} \int & d^{D_{k}} d^{D} q D_{\mu \rho}^{a b_{1}}(k) O_{\rho \nu}(k) D_{\nu \sigma}^{b b_{2}}(q) D^{c b_{3}}(q-k) \\
& \times G_{\mu \sigma \mu_{1} \cdots \mu_{A}}^{b_{1} b_{2} b_{3} a_{1} \cdots a_{E}}\left(k, q, q-k, p_{1}, \ldots, p_{E}\right),
\end{aligned}
$$

where $G_{\mu \sigma \mu_{1} \cdots \mu_{A}}^{b_{1} b_{2} b_{3} a_{1} \cdots a_{E}}\left(k, q, q-k, p_{1}, \ldots, p_{E}\right)$ is some appropriate Green function of the fields and their derivatives. The index $A$ denotes the number of external gauge fields, and $E=\sum_{i=1}^{6} n_{i}$ that of external momenta $p_{1}, p_{2}, \ldots, p_{E}$. Note that $E \geq 2$, since rigid invariance under $S U(N)$ and ghost number conservation imply that both $n_{1}+n_{2}$ and $n_{3}+n_{4}$ are larger than or equal to 1 . In what follows we prove that $I_{\mu_{1} \cdots \mu_{A}}^{a_{1} \cdots a_{E}}\left(p_{1}, \ldots, p_{E}\right)$ goes to zero as $D$ goes to 3 so that $B$ vanishes in this limit.

From eq. (2.23) it follows that every dimensionally regularized diagram contributing to the Green function (2.22) contains the contraction of the gauge propagator $D_{\mu \rho}^{a b_{1}}(k)$ with the operator $O_{\rho \nu}(k)$. Some trivial algebra yields (dropping colour indices):

$\hat{Y}_{\mu \nu}(k) \equiv D_{\mu \rho}(k) O_{\nu}^{\rho}(k)=\frac{m^{2}}{k^{2}\left(k^{2}+m^{2}\right)}\left(\hat{k}^{2} g_{\mu \nu}+\tilde{k}^{2} \hat{g}_{\mu \nu}+\hat{k}_{\mu} \hat{k}_{\nu}-\hat{k}_{\mu} k_{\nu}-k_{\mu} \hat{k}_{\nu}\right)$

and so the corresponding dimensionally regularized integrals only involve in their 
denominators factors of the type $\left(l^{2}\right)^{r_{1}}\left(l^{2}+m^{2}\right)^{r_{2}}$, with $r_{1}$ and $r_{2}$ non-negative integers. The operator $\hat{Y}_{\mu \nu}(p)$ is evanescent since it goes to zero as $D \rightarrow 3$ [43]. This does not mean, however, that the integrals contributing to $I_{\mu_{1} \cdots \mu_{A}}$, all of which contain one $\hat{Y}_{\mu \nu}(k)$, vanish as $D \rightarrow 3$. Indeed, integration over momenta could yield poles at $D=3$ that, conspired with the evanescent character of $\hat{Y}_{\mu \nu}(k)$, could give a non-vanishing result. A way to decide whether or not contributions containing evanescent operators go to zero as $D \rightarrow 3$ is to use the following property of dimensional regularization [43]: if

$$
K_{\mu_{1} \cdots \mu_{r}}(D) \equiv \int d^{D} k k_{\mu_{1}} \cdots k_{\mu_{r}} f(\tilde{k}, \hat{k}, p)
$$

is finite ${ }^{\star}$ at $D=3$ then

$$
\lim _{D \rightarrow 3} \hat{g}^{\mu_{1} \nu_{1}} \cdots \hat{g}^{\mu_{r} \nu_{r}} K_{\mu_{1} \cdots \mu_{r}}(D)=0 .
$$

A sufficient condition for $K_{\mu_{1} \cdots \mu_{r}}$ to be finite at $D=3$ is absolute convergence, which in turn can be established by using Lowenstein and Zimmerman's power counting theorem[44]. In applying this theorem some care should be taken and only scaling dimensions should be considered when counting powers. In other words, one should first express evanescent objects in terms of $D$-dimensional quantities contracted with $\hat{g}_{\mu \nu}$, e.g. $\hat{k}^{2}=\hat{g}^{\mu \nu} k_{\mu} k_{\nu}$, and secondly apply the theorem at $D=3$ prior to contractions.

Property (2.25) and the fact that all terms in $\hat{Y}_{\mu \nu}(k)$ have at least one $\hat{g}_{\mu \nu}$ imply that to prove that the breaking $B$ of the BRS identities approaches zero as $D \rightarrow 3$ it is enough to show that the integrals in (2.23) are finite at $D=3$. Let us see that this is the case. Consider a 1PI Feynman diagram contributing to (2.23),

\footnotetext{
* In the case at hand, the function $f$ in the integrand of $K_{\mu_{1} \cdots \mu_{r}}$ only depends on the $D$ dimensional vector $k^{\mu}$. The property is, however, more general and englobes the case in which $f$ is a function of $\tilde{k}^{\mu}$ and $\hat{k}^{\mu}$, which we will meet in Subsect. 2.2.
} 
hence to the Green function (2.22). Its superficial UV degree $\Omega$ at $D=3$ is given by

$$
\Omega=2-\frac{1}{2}\left[n_{1}+n_{2}+2\left(n_{3}+n_{4}+n_{6}\right)+3 n_{5}+V_{3}+V_{g h}+2 V_{4}\right] .
$$

Since $n_{1}+n_{2}, n_{3}+n_{4} \geq 1, \Omega$ satisfies the following inequality

$$
\Omega \leq \frac{1}{2}-\frac{1}{2}\left(V_{3}+V_{g h}+2 V_{4}+3 n_{5}+2 n_{6}\right) .
$$

For one-loop diagrams, the RHS in eq. (2.27) is in general positive, so that they are not finite by power counting. However, Speer [45] has shown that in dimensional regularization no poles arise in one-loop integrations for $D \rightarrow n$, ( $n$ odd) even when the integrals are not convergent by power counting. His results only apply to integrals whose denominators are products of the type $\left(p^{2}\right)^{r_{1}}\left(p^{2}+m^{2}\right)^{r_{2}}$, with $r_{1}$ and $r_{2}$ non-negative integers, which is why the simplification (2.24) has to be performed beforehand. Thus, one-loop integrals in (2.23) are free of singularities. As for higher-loop diagrams, they involve, at least, either two vertices of the type $V_{3}, V_{g h}$ and $V_{4}$ or two operators of the form $s A_{\mu}^{a}$ and $s c^{a}\left(n_{5}+n_{6} \geq 2\right)$ so that $\Omega<0$. The only possible sources of UV divergences beyond one loop are then subdiagrams. Now, one-loop subdiagrams are not a problem since they are finite by Speer's results. The situation is more complicated for two-loop subdiagrams, since 1PI two-loop diagrams contributing to the gauge field propagator are not UV finite by power counting [see discussion following eq. (2.13)]. However, as we shall show in Sect. 5 , their sum is finite for $D \rightarrow 3$ so that two-loop UV subdivergences cancel. To summarize, 1PI Feynman integrals in (2.23) do not give rise to UV poles as $D \rightarrow 3$. Obviously, the same statement holds for integrals which are not 1PI.

Let us finally see that no IR singularities occur in $I_{\mu_{1} \cdots \mu_{A}}$ as $D$ goes to 3 . IR singularities at $D=3$ may come either from integration over the regions

$$
k^{2} \sim 0, q^{2}>0 ; k^{2}>0, q^{2} \sim 0 ; k^{2} \sim 0, q^{2} \sim 0 ; \quad(k-q)^{2} \sim 0,
$$

or from subintegrations in $G_{\mu \sigma \mu_{1} \cdots \mu_{A}}^{b_{1} b_{2} b_{3} a_{1} \cdots a_{E}}\left(k, q, q-k, p_{1}, \ldots, p_{E}\right)$. Pisarski and Rao 
[5] have shown that the latter subintegrations do not give rise to IR singularities. In turn, from eq. (2.24) one may readily see that $\hat{Y}^{\mu \nu}(k) \sim$ const as $p$ goes to zero, and so, for IR divergences to take place at $D=3$, the behaviour of the Green function $G_{\mu \sigma \mu_{1} \cdots \mu_{A}}^{b_{1} b_{2} b_{3} a_{1} \cdots a_{E}}\left(k, q, q-k, p_{1}, \ldots, p_{E}\right)$ should be singular in one of the regions (2.28). Now, according to ref. [5], this only happens if all independent momenta in $G_{\mu \sigma \mu_{1} \cdots \mu_{A}}^{b_{1} b_{2} b_{3} a_{1} \cdots a_{E}}\left(k, q, q-k, p_{1}, \ldots, p_{E}\right)$ go to zero at the same time, but this is not possible in our case since we assume that the external momenta $p_{1}, \ldots, p_{E}$ are non-exceptional. This completes the proof of finiteness at $D=3$ of the integrals $I_{\mu_{1} \cdots \mu_{A}}$, which in turn implies the vanishing of the Green function (2.22), hence of the breaking $B$ so that BRS invariance is recovered when the limit $D \rightarrow 3$ is taken.

\subsection{BRS-invariant dimensional regularization of TMYM theory}

One may argue that it is desirable to preserve BRS invariance at the regularized level, since the BRS symmetry controls gauge invariance for the gauge-fixed theory. We next propose a method to dimensionally regularize TMYM theory which explicitly preserves BRS invariance. The starting point is the action $S_{m}^{n}[J, H]$ in eq. (2.16). The Feynman rules for this action are the same as in (2.6)-(2.10), except for the gauge propagator that now reads

$$
\begin{aligned}
\Delta_{\mu \nu}^{a b}(\tilde{p}, \hat{p})=\frac{\delta^{a b} m}{\left(p^{2}\right)^{2}+m^{2} \tilde{p}^{2}} & {\left[m \epsilon_{\mu \rho \nu} p^{\rho}+p^{2} g_{\mu \nu}-p_{\mu} p_{\nu}\right.} \\
& \left.+\frac{m^{2}}{p^{2}}\left(\frac{\hat{p}^{2}}{p^{2}} p_{\mu} p_{\nu}+\tilde{p}^{2} \hat{g}_{\mu \nu}-\hat{p}_{\mu} \hat{p}_{\nu}+p_{\mu} \hat{p}_{\nu}+\hat{p}_{\mu} p_{\nu}\right)\right] .
\end{aligned}
$$

The rest of the method proceeds along the same lines as before. With this new set of Feynman rules one first constructs $n$-dimensional diagrams. Then, one promotes $n$ to a complex variable $D$ so as to express Feynman integrals in terms of welldefined functions for $D$ in some domain of the complex plane. Finally, the value of the integrals outside the domain is obtained by analytic continuation. When constructing dimensionally regularized diagrams it must be taken into account that 
the denominator $\left(p^{2}\right)^{2}+m^{2} \tilde{p}^{2}$ makes it necessary to use dimensional regularization as defined in ref\$43,46,47], and not as in [40].

Let us see that the dimensionally regularized theory is BRS invariant. On the one hand, $n$-dimensional BRS transformations do not depend on $n$ explicitly and, as already mentioned, leave the action (2.16) invariant. On the other, since the propagators $\Delta_{\mu \nu}^{a b}(\tilde{p}, \hat{p}), D_{\mu}^{a b}(p)$ and $D^{a b}(p)$ have been computed by inverting the kinetic term in the action (2.16), the insertion of the operators $\epsilon_{\mu \rho \nu} \partial_{\rho} A_{\nu}^{a}-\partial_{\mu} b^{a}$, $\partial^{2} c^{a}$ and $\partial^{2} \bar{c}^{a}$ in a line of a dimensionally regularized diagram is equivalent to the contraction of the line to a point. From these two facts it follows [40] that the dimensionally regularized theory is consistent with an action principle and that, therefore, the corresponding Green functions satisfy the formal BRS identities.

The non-trivial dependence of the gauge field propagator (2.29) on $\tilde{p}^{\mu}$ and $\hat{p}^{\mu}$ makes calculations very complicated. However, when the limit $D \rightarrow 3$ is taken, the set of Feynman rules (2.29) and (2.7)-(2.11) generate the same perturbation expansions as the set (2.6)-(2.11). Here we will show that this is the case at one loop by using property (2.25), and then recall general results from renormalization theory to extend the result to higher loops. To state the problem more clearly it is convinitent to write $(2.29)$ as the sum

$$
\Delta_{\mu \nu}^{a b}(\tilde{p}, \hat{p})=D_{\mu \nu}^{a b}(p)+R_{\mu \nu}^{a b}(\tilde{p}, \hat{p})
$$

with $D_{\mu \nu}^{a b}(p)$ as in eq. (2.6) and $R_{\mu \nu}^{a b}(\tilde{p}, \hat{p})$ the evanescent operator

$$
\begin{gathered}
R_{\mu \nu}^{a b}(\tilde{p}, \hat{p})=\frac{m^{3}}{p^{2}\left[\left(p^{2}\right)^{2}+m^{2} \tilde{p}^{2}\right]}\left[\frac{\hat{p}^{2}}{p^{2}+m^{2}}\left(\epsilon_{\mu \rho \nu} p^{\rho}+p^{2} g_{\mu \nu}+\frac{m^{2}}{p^{2}} p_{\mu} p_{\nu}\right)\right. \\
\left.+\tilde{p}^{2} \hat{g}_{\mu \nu}+\hat{p}_{\mu} \hat{p}_{\nu}-p_{\mu} \hat{p}_{\nu}-\hat{p}_{\mu} p_{\nu}\right] .
\end{gathered}
$$

Then, any 1PI Feynman diagram $\mathcal{F}(D)$ with $I_{g}$ internal gauge lines can be recast 
as

$$
\mathcal{F}(D)=\sum_{j=0}^{I_{g}} \mathcal{F}_{j}(D)
$$

where $\mathcal{F}_{j}(D)$ is the sum of all terms with $j$ evanescent operators $R_{\mu \nu}^{a b}$ and $I_{g}-j$ factors $D_{\mu \nu}^{a b}$. In particular, $\mathcal{F}_{0}(D)$ is obtained from $\mathcal{F}(D)$ by replacing the full propagator (2.29) with the simpler (2.6) in all internal gauge lines. Now, any 1PI Green function $\mathcal{G}(D)$ is obtained by summing over all topologically non-equivalent 1PI Feynman diagrams with appropriate symmetry factors. Thus, $\mathcal{G}(D)$ can be written as

$$
\mathcal{G}(D)=\mathcal{G}_{0}(D)+\hat{\mathcal{G}}(D)
$$

with $\mathcal{G}_{0}(D)$ the Green function as obtained from the Feynman rules (2.6)-(2.11). The aim is to show that $\hat{\mathcal{G}}(D)$ goes to zero as $D$ appraoches 3 .

We begin by using property (2.25) to show that at one loop, $\mathcal{F}_{j}(D) \rightarrow 0$ as $D \rightarrow 3$ for $j \geq 1$, which in turn implies that $\hat{\mathcal{G}}(D)$ goes to zero as $D$ approaches 3. Property (2.25) requires finiteness at $D=3$ so a detailed analysis of the convergence properties of $\mathcal{F}_{j}(D)$ is in order. The UV degree of $\Delta_{\mu \nu}^{a b}(\tilde{p}, \hat{p})$ is the same as that of $D_{\mu \nu}^{a b}(p)$, namely -2 , - the degree used to compute $\omega_{m}$ in eq. (2.13). In turn, $R_{\mu \nu}^{a b}(\tilde{p}, \hat{p})$ has UV degree -4 , i.e. two units less, and so the overall UV degree of $\mathcal{F}_{j}(D)$ at $D=3$ is $\omega_{m}-2 j$. Diagrams with only one external gauge leg vanish because of colour algebra (see Sect. 4). Also, there are no diagrams with only one external ghost leg, since they would violate ghost conservation number. Using then that diagrams with two or more external legs have $\omega_{m} \leq 1$ we conclude that $\mathcal{F}_{j}(D)(j \geq 1)$ is overall UV convergent at $D=3$. As for IR convergence, the IR degrees of $\Delta_{\mu \nu}^{a b}(\tilde{p}, \hat{p}), D_{\mu \nu}^{a b}(p)$ and $R_{\mu \nu}^{a b}(\tilde{p}, \hat{p})$ are respectively $-2,-1$ and -2. The IR behaviour of $\mathcal{F}_{j}(D)$ for all $j$ is then at least as good as that of $\mathcal{F}(D)$, and thus if the full diagram $\mathcal{F}(D)$ is overall IR finite by power counting so are all the terms $\mathcal{F}_{j}(D)$. Putting everything together, we have that if a one-loop diagram does not have IR divergences, property (2.25) implies that $\lim _{D \rightarrow 3} \mathcal{F}_{j}(D)=0$ 
for $j \geq 1$. Now, power counting shows that one-loop diagrams are IR convergent at $D=3$ for non-exceptional external momenta, something very easy to check for the diagrams we are going to be concerned with (see. Figs. 2, 7 and 9). It then follows that for one-loop diagrams, $\mathcal{F}_{j \geq 1}(D)$ goes to zero as Dto3 and that we can use (2.6) as the gauge field propagator to obtain their non-vanishing contributions in this limit. Therefore, as $D$ approaches 3, one-loop diagrams only involve denominators of the type $\left(p^{2}\right)^{r_{1}}\left(p^{2}+m^{2}\right)^{r_{2}}$, with $r_{1}$ and $r_{2}$ non-negative integers, Speer's [45] conclusions apply and, as a result, one-loop diagrams are finite at $D=3$.

To show that $\hat{\mathcal{G}}(D)$ vanishes as $D \rightarrow 3$ at the two-loop order we shall take advantage of two general results from perturbative renormalization[43,48]. Let us consider a 1PI Green function that is UV divergent at the $(n+1)$-loop order and that has been renormalized up to order $n$. The first result states that the UV divergent part of the function at order $n+1$ is a local polynomial in the external momenta, with degree at most equal to the superficial UV degree of the function at $n+1$ loops. Let us state the second result. We shall consider the renormalized values of a 1PI Green function obtained by using two different renormalization schemes and assume that these values are equal up to the $n$-loop order. Then, their difference at order $n+1$ is a polynomial in the external momenta if the superficial UV degree of the function at $n+1$ loops is equal or larger than zero. The degree of this polynomial is at most equal to the UV degree of the function at $n+1$ loops. If the superficial UV degree of the function at $n+1$ loops is negative, then the difference vanishes.

We have seen that both dimensional regularization methods introduced in this and the previous subsections yield a finite limit as $D \rightarrow 3$ at one loop. These limits define therefore two renormalization schemes at the one-loop order. Moreover, these two renormalization schemes agree at this order, since we have seen that both $D \rightarrow 3$ limits are equal at one loop. Hence, the two results above ensure that the limit $D \rightarrow 3$ of any 1PI function, but for the vacuum polarization tensor, is finite at the two-loop level and that it is the same for both dimensional regularization 
methods, i.e. $\hat{\mathcal{G}}(D) \rightarrow 0$ as $D \rightarrow 3$.

The two-loop vacuum polarization tensor is logarithmically overall UV divergent. Thus, the difference between the vacuum polarization tensor in the regularization of Subsect. 2.1, $\Pi_{\mu \nu}^{(2.1)}$, and the vacuum polarization tensor for the regularization introduced in the present subsection, $\Pi_{\mu \nu}^{(2.2)}$, is

$$
\Pi_{\mu \nu}^{(2.1)}-\Pi_{\mu \nu}^{(2.2)}=m\left(a_{1} \tilde{g}_{\mu \nu}+a_{2} \hat{g}_{\mu \nu}\right)+O(D-3)
$$

A number of comments regarding eq. (2.32) are in order. First, the overall factor $m$ occurs since the mass dimension of the vacuum polarization tensor is one, despite its superficial UV degree is zero. Secondly, the fact that the coefficients $a_{1}$ and $a_{2}$ might not be equal is a consequence of the fact that our definition for the $n$ dimensional $\epsilon_{\mu \rho \nu}$ renders the formal theory invariant under $S O(3) \otimes S O(n-3)$ rather than $S O(n)$ [41]. Finally, we have not made any hypothesis on the finiteness of $a_{1}$ and $a_{2}$ as $D \rightarrow 3$. Now, we have seen that both dimensional regularization methods yield BRS-invariant 1 PI functions as $D \rightarrow 3$. Hence, in the limit $D \rightarrow 3$ we have:

$$
p^{\mu} \Pi_{\mu \nu}^{(2.1)}=p^{\mu} \Pi_{\mu \nu}^{(2.2)}=0
$$

Eqs. (2.32) and (2.33) lead to

$$
a_{1}=a_{2}=0
$$

and so, $\hat{\mathcal{G}}(D)$ goes to zero as $D$ approaches 3 for the two-loop vacuum polarization tensor. We have then shown that the two-loop vacuum polarization tensor computed with any of the regularization methods in this section is the same, modulo contributions that vanish in the limit $D \rightarrow 3$. It is worth noticing that the general arguments we have used to show that at two loops $\Pi_{\mu \nu}^{(2.1)}-\Pi_{\mu \nu}^{(2.2)}$ goes to zero as $D \rightarrow 3$ would have left behind a non-vanishing contribution of the type

$$
a_{3} \epsilon_{\mu \rho \nu} p^{\rho}
$$

had we applied them at the one-loop level. 
Let us analyze now what happens beyond the two-loop order. Now the superficial UV degree of any 1PI function of TMYM theory is negative. We shall see in Sect. 5 that the limit $D \rightarrow 3$ of the two-loop vacuum polarization tensor is actually finite. Then, putting everything together, we conclude that the limit $D \rightarrow 3$ of any 1PI function of TMYM theory is finite at $n$ loops. Moreover, the latter limit is the same for both regularization methods in this section. This shows UV finiteness for TMYM theory at any order in the loop expansion (see also Sect 7).

To summarize, only the non-evanescent part (2.6) of the gauge field propagator (2.29) contributes to the limit $D \rightarrow 3$ of 1 PI Green functions, the objects we will compute here. It is important to emphasize, however, that the starting point is the full propagator (2.29), which ensures BRS invariance at the regularized level, and that it happens a posteriori that as $D$ goes to 3 only its non-evanescent part contributes.

Notice that for any of the two dimensional regularizations we have defined, the 3-dimensional limit of dimensionally regularized TMYM theory is BRS-invaraint. Once this has been established, it follows that the limit $m \rightarrow \infty$ preserves BRS invariance, since the regulator $m$ does not enter explicitly in the BRS identities.

Here we adopt the BRS-invariant definition of dimensional regularization of this subsection. In the remainder of the paper, dimensionally regularized quantities should be understood to be defined in this sense. As far as explicit computations are concerned, both approaches give the same result. However, from the point of view of understanding the results, they provide different pictures, as will become clear in Sect. 5.

In this paper we are going to evaluate the limit $D \rightarrow 3, m \rightarrow \infty$ of some dimensionally regularized TMYM Green functions. As is by now clear, their 3dimensional limit only receives contributions from non-evanescent objects so that to compute the relevant Feynman diagrams we will use (2.6) for the gauge field propagator. As for the large $m$ limit, we present in the next section two vanishing 
theorems that will be very helpful in computing it.

\section{Large mass vanishing theorems}

In this section we present two power counting-like theorems which provide sufficient conditions for an integral arising from a Feynman diagram to vanish in the limit $m \rightarrow \infty$. These theorems will be widely used in Sects. 5 and 6 below. They will enable us not only to disregard integrals that do not contribute to the limit $m \rightarrow \infty$ without having to compute them, but also to explicitly evaluate non-zero contributions.

In what follows we will be concerned with integrals of the form

$$
I(p, m)=m^{\beta} \int d k F(k, p, m)
$$

where the integration measure is

$$
d k=d^{N} k_{1} \cdots d^{N} k_{M}
$$

and the integrand is a rational function:

$$
F(k, p, m)=\frac{M(k)}{\prod_{i \in H}\left(l_{i}^{2}+m_{i}^{2}\right)^{n_{i}}} .
$$

The greek letter $\beta$ denotes an arbitrary real number and $N$ and $n_{i}$ are positive integers. The numerator $M(k)$ is a monomial of degree $n_{k}$ in the components of the vectors $k_{1}, \ldots, k_{M}$. The $l_{i}$ 's are linear combinations

$$
\begin{gathered}
l_{i}=K_{i}(k)+P_{i}(p) \\
K_{i}(k)=\sum_{j=1}^{M} a_{i j} k_{j} \quad, \quad P_{i}(p)=\sum_{j=1}^{E} b_{i j} p_{j},
\end{gathered}
$$

with not all the coefficients $a_{i j}$ vanishing for any given $i$. We will assume that the external momenta $p_{1}, \ldots, p_{E}$ lie in a bounded subdomain of $\mathbb{R}^{D}$ and consider the 
case in which the masses only take on two values, namely $m_{i}=0$ for, say, $i \in S_{0}$ and $m_{i}=m>0$ for $i \in S_{1}=H \backslash S_{0}$. We want to find sufficient conditions for the integral (3.1) to go to zero as $m \rightarrow \infty$ so that we can assume without loss of generality that $m / p>1$.

We call $d$ to the mass dimension of $I(p, m)$ and denote by $\underline{\omega}_{\text {min }}$ the minimum of zero and the infrared degrees of all the subintegrals of $I(p, m)$ at zero external momenta, including $I(p, m)$ itself. Then the following theorem holds:

$m$-Theorem: If the integral $I(p, m)$ is both $U V$ and IR covergent by power counting at non-exceptional external momenta, and the mass dimension $d$ and $\underline{\omega}_{\min }$ defined above satisfy

$$
d-\underline{\omega}_{\min }<0
$$

then $I(p, m)$ vanishes when $m$ goes to $\infty$.

To prove the theorem we will proceed in two steps. We will first bound $I(p, m)$ from above keeping $m / p>1$ fixed, the bound in general depending on $m$, and then find conditions for the bound to approach zero as $m \rightarrow \infty$.

Following Lowenstein and Zimmermann [44] we write the integral (3.1) as

$$
I(p, m)=\sum_{S} I_{S}(p, m)
$$

where

$$
I_{S}(p, m)=m^{\beta} \int_{\substack{l_{i}^{2}<m^{2} \\ l_{i}^{2} \geq m^{2}}} d k F(k, p, m)
$$

and the sum is extended to all subsets $S$ of $S_{0}$. $T$ is the complement of $S$ in $S_{0}$, i.e. $T=S_{0} \backslash S$. To study $I_{S}(p, m)$, we note that among the $l_{i}$ 's occuring in the denominator of (3.2) we can choose without loss of generality $M$ of them such 
that the change of variables

$$
\left(k_{1}, \ldots, k_{M}\right) \longrightarrow\left(u_{1}=l_{i_{1}}, \ldots, u_{a}=l_{i_{a}}, v_{1}=l_{i_{a+1}}, \ldots, v_{b}=l_{i_{M}}\right)
$$

has jacobian one. Furthermore, $l_{i_{1}}, \ldots, l_{i_{M}}$ can be chosen in such a way that the homogeneous parts in $k_{i}$ of $u_{1}, \ldots, u_{a}$ form a basis of the homogeneous parts in $k_{i}$ of the $l_{j}$ 's, $j \in S$. In terms of the new variables, the numerator $M(k)$ in $(3.2)$ reads

$$
M(k)=\sum_{\alpha} A_{S \alpha}(v, p) M_{\alpha}(u)
$$

with $M_{\alpha}(u)$ a monomial in the components of $u_{1}, \ldots, u_{a}$ and $A_{S \alpha}(v, p)$ a polynomial in those of $v_{1}, \ldots, v_{b}$ and the external momenta $p_{1}, \ldots, p_{E}$. As for the vectors $l_{i}$, we have that

$$
\begin{gathered}
l_{i}=U_{i}(u)+Q_{i}(p) \quad \text { if } \quad i \in S, \\
l_{i}=V_{i}(v)+R_{i}(u, p) \quad \text { if } \quad i \notin S,
\end{gathered}
$$

with $U_{i}, Q_{i}, V_{i}$ and $R_{i}$ linear combinations of their arguments. For the integral $I_{S}(p, m)$ we obtain

$$
I_{S}(p, m)=m^{\beta} \sum_{\alpha} \int_{l_{i}^{2}<m^{2}} d u \frac{M_{\alpha}(u)}{\prod_{S}\left(l_{i}^{2}\right)^{n_{i}}} \int_{l_{i}^{2} \geq m^{2}} d v \frac{A_{S \alpha}(v, p)}{\prod_{T}\left(l_{i}^{2}\right)^{n_{i}} \prod_{S_{1}}\left(l_{i}^{2}+m^{2}\right)^{n_{i}}} .
$$

Note that when integrating over $v$, the $R_{i}$ 's play the rôle of bounded external momenta, since the vectors $u_{i}$ and $p_{i}$ are themselves bounded. From this observation, the fact that $p / m<1$ and proceeding in the same way as in [44] we get that

$$
I_{S}(p, m) \leq \sum_{\alpha} C_{S \alpha} m^{d-d_{S \alpha}} I_{S \alpha}(p, m)
$$

where $\left\{C_{S \alpha}\right\}$ are constants that do not depend on $m$, and $d$ and $d_{S \alpha}$ are the 
mass dimensions of the integrals $I(p, m)$ and

$$
I_{S \alpha}(p, m)=\int_{l_{i}^{2}<m^{2}} d u \frac{\left|M_{\alpha}(u)\right|}{\prod_{S}\left(l_{i}^{2}\right)^{n_{i}}}
$$

We thus have that

$$
I(p, m) \leq \sum_{S, \alpha} C_{S \alpha} m^{d-d_{S \alpha}} I_{S \alpha}(p, m)
$$

Note that $I_{S \alpha}(p, m)$ depends on $m$ through the domain of integration. Moreover, $m$ is an UV cut-off for the integration variables $u_{i}$ so that the integration domain is bounded. The integrals $I_{S \alpha}(p, m)$ being then covergent at non-exceptional external momenta. To estimate the large $m$ behaviour of $I(p, m)$ we have thus been led to evaluate the behaviour of the massless integrals $I_{S \alpha}(p, m)$ as their $\mathrm{UV}$ cut-off $m$ goes to infinity. The latter is usually done by using Weinberg's theorem[49], which establishes that the leading contribution to $I_{S \alpha}(p, m)$ as $m \rightarrow \infty$ is bounded from above by

$$
C_{S \alpha}^{\prime} m^{\bar{\omega}_{S \alpha}^{\max }}[\ln (m / p)]^{L_{S \alpha}}
$$

Here $C_{S \alpha}^{\prime}$ is a positive constant, $L_{S \alpha}$ is a natural number and $d_{S \alpha}^{\max }$ is given by

$$
\bar{\omega}_{S \alpha}^{\max }=\max \left\{0,\left\{\bar{\omega}_{i}\right\}\right\}
$$

where the $\bar{\omega}_{i}$ 's are the UV degrees of all the subintegrals of $I_{S \alpha}(p, m)$ including itself. Notice that if all the $\bar{\omega}_{i}$ 's are negative, the integral $I_{S \alpha}(p, m)$ is convergent and the bound (3.4) holds with $L_{S \alpha}$ zero.

Let us mention that the fact that Weinberg's theorem can be applied to determine the large $m$ behaviour of $I_{S \alpha}(p, m)$ involves some technical subtleties. Indeed, the integrals we are studying are massless, whereas Weinberg considered 
massive integrals so that his techniques did not fall short of mathematical rigour. For this reason we have rigourously checked that if $I(p, m)$ in eq. (3.1) is either a one- or a two-loop integral, the large $m$ leading contribution from any of its $I_{S \alpha}(p, m)$ is indeed bounded from above by (3.4). We did so by using the methods in [44], suitable for massless integrals. To avoid an unnecessarily long paper, we will not present the details here. These techniques can be applied to any arbitrary integral of the type $I_{S \alpha}(p, m)$. We thus strongly believe that the bound provided by eq. (3.4) holds whatever the number of loops. Let us also stress that in this paper we are only concerned with one- and two-loop integrals. After these remarks, we come to the following result:

$$
\lim _{m \rightarrow \infty} I(p, m) \leq \lim _{m \rightarrow \infty} \sum_{S, \alpha} C_{S \alpha}^{\prime \prime} m^{\left(d-d_{S \alpha}+\bar{\omega}_{S \alpha}^{\max }\right)}[\ln (m / p)]^{L_{S \alpha}}
$$

Next we note that the UV degree of the integrals $I_{S \alpha}(p, m)$ is equal to minus their IR degree at zero external momenta, these integrals being massless. It then follows that

$$
d_{S \alpha}-\bar{\omega}_{S \alpha}^{\max }=\min \left\{0, \underline{\omega}_{S \alpha}^{\min }\right\}
$$

where $\underline{\omega}_{S \alpha}^{\min }$ is the minimum of the IR degrees at zero momenta of $I_{S \alpha}$ and all its subintegrals. Finally,

$$
\min \left\{d_{S \alpha}-\bar{\omega}_{S \alpha}^{\max }\right\}=\min \left\{0,\left\{\underline{\omega}_{S \alpha}^{\min }\right\}\right\}=\underline{\omega}_{\min },
$$

where the minimum is computed over the set of all $(S, \alpha)$. Putting everything together, we obtain

$$
\lim _{m \rightarrow \infty} I(p, m) \leq \lim _{m \rightarrow \infty}\left[C^{\prime \prime \prime} m^{\left(d-\underline{\omega}_{\min }\right)}[\ln (m / p)]^{\max \left\{L_{S \alpha}\right\}}\right]
$$

so that

$$
\lim _{m \rightarrow \infty} I(p, m)=0
$$

if $d-\underline{\omega}_{\min }<0$. This completes the proof of the theorem. 
To formulate the second theorem announced at the beginning of the section we remind that $n_{k}$ denotes the degree in $k$ of the numerator in $I(p, m)$ and introduce the symbol

$$
[n]= \begin{cases}1 & \text { for } n \text { odd } \\ 0 & \text { for } n \text { even } .\end{cases}
$$

The theorem states the following:

o-Theorem: If the integral $I(p, m)$ in (3.1) is absolutely convergent at zero external momenta and its mass dimension $d$ satisfies

$$
\left[n_{k}\right]>d,
$$

then $I(p, m) \rightarrow 0$ as $m \rightarrow \infty$.

The hypothesis about convergence is more restrictive if compared to that of the $m$-theorem, since now IR convergence for zero external momenta is required. Nevertheless, the theorem covers cases which escape the $m$-theorem and in this sense is complementary to the latter.

As for the proof, let us start by rescaling the integrated momenta $k \rightarrow k m$. Eq. (3.1) thus becomes

$$
I(p, m)=m^{d} J(p / m)
$$

where

$$
J(p / m)=\int d k F(k, p / m, 1) .
$$

Since the limit $m \rightarrow \infty$ of $J(p / m)$ is well defined by the hypothesis of abolute convergence at zero external momenta, we have that

$$
\lim _{m \rightarrow \infty} I(p, m)=J(0) \lim _{m \rightarrow \infty} m^{d}
$$

Now, condition (3.5) implies that $d \leq 0$ so that the only case for which the RHS in (3.6) is not zero trivially is $d=0$, which in turn corresponds to $n_{k}$ odd. But for $n_{k}$ odd, $J(0)$ is zero by $S O(N)$ covariance. This closes the proof of the theorem. 
We finish this section with a remark about the hypothesis of UV convergence in the $m$-theorem as applied to TMYM theory. Let us recall that we are seeking for results that allow us to establish whether a dimensionally regularized integral vanish in the limit $D \rightarrow 3, m \rightarrow \infty$. The most general dimensionally regularized integral that we will find in the next sections is of the form

$$
I(p, m)=m^{\beta} \int d^{D} k d^{D} q F(k, q, p, m)
$$

where $k$ and $q$ are the internal momenta and $p$ stands, as usual, for the external momenta $p_{1}, \ldots, p_{E}$. The integrand is

$$
F(k, q, p, m)=\frac{k^{n_{k}} q^{n_{q}}}{\prod_{A}\left[K_{i}^{2}\left(K_{i}^{2}+m_{i}^{2}\right)\right]^{n_{i}} \prod_{B}\left[Q_{i}^{2}\left(Q_{i}^{2}+m_{i}^{2}\right)\right]^{n_{i}} \prod_{C}\left[R_{i}^{2}\left(R_{i}^{2}+m_{i}^{2}\right)\right]^{n_{i}}}
$$

with $K_{i}$ a linear combination of $k$ and $p, Q_{i}$ of $q$ and $p$, and $R_{i}$ of $k, q$ and $p$. We shall assume in the sequel that $I(p, m)$ is IR convergent by power counting at non-exceptional external momenta and $D=3$, as is the case for the two-loop integrals we will find. Regarding UV convergence, it demands overall convergence and convergence along the $k$ - and $q$-loops, or more precisely:

$$
\begin{gathered}
\bar{\omega} \equiv 6+n_{k}+n_{q}-4 \sum_{A, B, C} n_{i}<0 \\
\bar{\omega}_{k} \equiv 3+n_{k}-4 \sum_{A, C} n_{i}<0 \\
\bar{\omega}_{q} \equiv 3+n_{q}-4 \sum_{B, C} n_{i}<0
\end{gathered}
$$

where we have taken $D=3$. These conditions are not always met in our case, since power counting as applied to TMYM theory yields the following two situations:

$$
\begin{array}{ll}
\bar{\omega}<0, & \bar{\omega}_{k}=0,1, \quad \bar{\omega}_{q}<0, \\
\bar{\omega}<0, & \bar{\omega}_{k}<0,
\end{array}
$$

In both scenarios the integral is overall UV convergent by power counting but not along one of its subintegrals. In our TMYM computations, $\bar{\omega}_{k}$ and $\bar{\omega}_{q}$ are never 
larger than one. Notice that if $\bar{\omega}<0$, then both $\bar{\omega}_{k}$ and $\bar{\omega}_{q}$ cannot be equal or larger than zero at the same time. Now, we know that in dimensional regularization one-loop integrals of the type we are considering are free of singularities as $D$ goes to 3 [45]. This makes us suspect that conditions (3.9) and (3.10) requiring absence of UV subdivergences could be dropped. Let us show that this is so. Here we present the proof for the case in which $\bar{\omega}_{k}=0,1$.

To be more specific, we want to prove that as far as the requirement of UV convergence is concerned, it is enough to demand (3.8), even when $\bar{\omega}_{k}=0,1$, to rightly apply the $m$-theorem in the finite limit $D \rightarrow 3$. To do this we write $F(k, q, p, m)$ as

$$
F(k, q, p, m)=E(k, q, p, m) G(q, p, m),
$$

$E$ and $G$ being given by

$$
E(k, q, p, m)=\frac{k^{n_{k}}}{\prod_{A}\left[K_{i}^{2}\left(K_{i}^{2}+m_{i}^{2}\right)\right]^{n_{i}} \prod_{C}\left[R_{i}^{2}\left(R_{i}^{2}+m_{i}^{2}\right)\right]^{n_{i}}}
$$

and

$$
G(q, p, m)=\frac{q^{n_{q}}}{\prod_{B}\left[Q_{i}^{2}\left(Q_{i}^{2}+m_{i}^{2}\right)\right]^{n_{i}}} .
$$

We further decompose $E$ as

$$
E(k, q, p, m)=E_{\text {fin }}^{(l)}(k, q, p, m)+E_{\text {div }}^{(l)}(k, m),
$$

where $l=\bar{\omega}_{k}$ and

$$
\begin{gathered}
E_{\mathrm{fin}}^{(l)}(k, q, p, m)=\left[1-t^{(l)}(q=0, p=0)\right] E^{(l)}(k, q, p, m), \\
E_{\operatorname{div}}^{(l)}(k, q, m)=t^{(l)}(q=0, p=0) E^{(l)}(k, q, p, m) .
\end{gathered}
$$


The operators $t^{(l)}$ read:

$$
\begin{aligned}
& t^{(0)}(q=0, p=0) H(k, q, p)=H(k, 0,0) \\
& t^{(1)}(q=0, p=0) H(k, q, p)=H(k, 0,0)+q^{\mu}\left[\frac{\partial H(k, q, p)}{\partial q^{\mu}}\right]_{q=p=0}+p^{\mu}\left[\frac{\partial H(k, q, p)}{\partial p^{\mu}}\right]_{q=p=0}
\end{aligned}
$$

Note that by construction $E_{\text {fin }}^{(l)}$ carries the UV finite part and $E_{\text {div }}^{(l)}$ the divergent (or singular) one. As a matter of fact, $E_{\text {fin }}^{(l)}$ is a BPHZ subtraction at zero momentum $q=0, p=0[50]$. The decomposition (3.11) gives for $I(p, m)$ :

$$
I(p, m)=I_{\text {fin }}^{(l)}(p, m)+I_{\operatorname{div}}^{(l)}(p, m),
$$

with

$$
\begin{gathered}
I_{\text {fin }}^{(l)}(p, m)=m^{\beta} \int d^{D} k d^{D} q E_{\text {fin }}^{(l)}(k, q, p, m) G(q, p, m), \\
I_{\text {div }}^{(l)}(p, m)=m^{\beta} \int d^{D} k d^{D} q E_{\text {div }}^{(l)}(k, q, m) G(q, p, m) .
\end{gathered}
$$

The integral $I_{\text {fin }}^{(l)}(p, m)$ is already UV convergent by power counting at $D=3$. Regarding its IR convergence, we have to make sure that the zero-momentum subtractions we have performed do not introduce any IR divergences. Since the subtractions are at zero momenta, we have to analyze the power counting concerning integration over the regions $k \sim 0, q \sim 0$, and $k \sim 0, q \sim 0$. The IR degree $\underline{\omega}_{k}$ of $I_{\text {fin }}^{(l)}(p, m)$ in the region $k \sim 0$ satisfies

$$
\underline{\omega}_{k} \geq 3+n_{k}-2 \sum_{A, C} n_{i}-l .
$$

Taking into account that $l=\bar{\omega}_{k}=3+n_{k}-4 \sum_{A, C} n_{i}$, we conclude that

$$
\underline{\omega}_{k} \geq 2 \sum_{A, C} n_{i}>0
$$

Thus, IR finiteness holds by power counting for the $k$-integration in $I_{\text {fin }}^{(l)}(p, m)$ at non-exceptional external momenta and $D=3$. IR finiteness at non-expectional 
momenta for any other domain of $I_{\text {fin }}^{(l)}(p, m)$ holds as well, since $I(p, m)$ is IR finite by power counting at non-exceptional momenta and $D=3$. This shows that $I_{\text {fin }}^{(l)}(p, m)$ is both UV and IR convergent by power counting at non-exceptional momenta and $D=3$, which in turn implies that the limit $D \rightarrow 3$ can be taken inside the integral. As for the other conditions required by the $m$-theorem for $I_{\text {fin }}^{(l)}(p, m)$ to vanish as $m$ goes to $\infty$, it is easy to see that they follow from those for $I(p, m)$. Concerning $I_{\text {div }}^{(l)}(p, m)$, some simple algebra shows that it is a linear combination of products of two one-loop integrals. The integrals over $k$ are independent of $p$ and their dependence on $m$ can be scaled away by rescaling the integration variable $k \rightarrow k m$. This confines the initial UV divergence to integrals independent of $m$ and $p$ which in the limit $D \rightarrow 3$ will produce finite constants $\mathcal{K}_{\alpha}[45]:$

$$
\begin{gathered}
I_{\operatorname{div}}^{(l)}(p, m)=\sum_{\alpha=0}^{l} \mathcal{K}_{\alpha} \int d^{3} q \frac{m^{2 \alpha} q^{\alpha+n_{q}}}{\prod_{B}\left[Q_{i}^{2}\left(Q_{i}^{2}+m_{i}^{2}\right)\right]^{n_{i}}} \\
\mathcal{K}_{\alpha}=\lim _{D \rightarrow 3} \int d^{D} k k^{n_{k}+\alpha} f_{\alpha}\left(k^{2}\right)
\end{gathered}
$$

with $f_{\alpha}\left(k^{2}\right)$ known functions of $k^{2}$. Integrals over $q$ in (3.13) are UV convergent, since their UV degree is $3+\alpha+n_{q}-2 \sum_{B} n_{i}=\alpha-l+\bar{\omega}$ and $\bar{\omega}<0$ by hypothesis. They are also IR convergent at non-exceptional external momenta by hypothesis. Thus we can use the $m$-theorem to study their large $m$ limit. Again, it is a matter of algebra to show that the conditions that the $m$-theorem demands for (3.13) to vanish as $m \rightarrow \infty$ follow from those for $I(p, m)$, with the exception of $\bar{\omega}_{k}=l<0$, which now is no longer needed.

All in all, we have that the integral (3.7) approaches zero as $m$ goes to $\infty$ if all the conditions demanded by the $m$-theorem, except UV convergence along $k$, are met. It is straightforward to see that the same methods show that UV covergence along $k$ can also be dropped for the $o$-theorem. 


\section{The one-point Green function for the gauge field}

Let us consider the gauge field one-point Green function $<A_{\mu}^{a}(x)>$ for TMYM theory with action (2.4). According to eq. (2.13), the integrals that occur in 1PI Feynman diagrams with only one external gauge line and no external ghost lines

have the highest superficial UV degree. In fact, one has to go to order $g^{6}$ to get superficial UV convergence by power counting. Dimensional regularization, as defined in any of the two forms in Sect. 2, renders these divergent diagrams finite. Likewise, IR divergences at non-exceptional external momenta are regularized. It thus makes sense to speak of the corresponding dimensionally regularized diagrams.

Every single dimesionally regularized 1PI Feynman diagram that occurs in the computation of $\left\langle A_{\mu}^{a}(x)\right\rangle$ is zero due to colour algebra. Indeed, its colour factor can always be recast as a linear combination of terms of the form

$$
\operatorname{Tr}\left(t^{a} t^{a_{1}} \cdots t^{a_{i}}\right) \operatorname{Tr}\left(t^{a_{i+1}} \cdots t^{a_{j}}\right) \cdots \operatorname{Tr}\left(t^{a_{k}} \cdots t^{a_{2 N}}\right)=C^{a},
$$

where all colour indices $a_{1} \cdots a_{2 N}$ are contracted in pairs and the $t^{a}$ denote the generators in the adjoint representation of $S U(N)$. Obviously, $C^{a}=0$. This implies that $(i)$ diagrams with gauge field one-point subdiagrams are zero and that (ii) the Green function $\left\langle A_{\mu}^{a}(x)\right\rangle$ vanishes. The latter is the same as to say that there are no contributions to the TMYM regularized effective action with only one gauge-field external leg. By taking then into account the definition in eq. (2.12) one concludes that the effective action for perturbative CS field theory has no one-point term for the gauge field. 


\section{The gauge field vacuum polarization tensor}

The purpose of this section is to describe how we have carried out the computation of the vacuum polarization tensor $\Pi_{\mu \nu}^{a b}(p)$ for $S U(N)$ CS theory in the Landau gauge at second order in perturbation theory. This entails as a first step the calculation of the limit $D \rightarrow 3$ of the dimensionally regularized topologically massive $S U(N)$ two-loop vacuum polarization tensor $\Pi_{\mu \nu}^{a b}(p, m, D)$, obtained by adding the diagrams in Figs. 3-6. The second step is to compute the large $m$ limit of the corresponding 3-dimensional result. We shall see that both limits $D \rightarrow 3$ and $m \rightarrow \infty$ exist when taken them in this prescribed order and once we have summed over all Feynman diagrams. This is in agreement with refs. [27] and [28], where finiteness of CS theory in the Landau gauge is proved at any order in pertubation theory. Let us also point out that in accordance with the regularization

method explained in Sect. 2, $\Pi_{\mu \nu}^{a b}(p, m, D)$ is the fully regularized CS vacuum polarization tensor.

At first order in perturbation theory there are three diagrams that contribute to the polarization tensor (see Fig. 2). Their value was given in [5] for finite $m$, and the large $m$ limit was independently computed in[21,22,51]:

$$
\Pi_{\mu \nu}^{(1) a b}(p)=-\frac{7}{3} \frac{g^{2} c_{V}}{4 \pi} \delta^{a b} \epsilon_{\mu \nu \rho} p^{\rho} .
$$

Here we will focuss on the two-loop correction.

We have shown in Sect. 2 that the non-vanishing contributions as $D \rightarrow 3$ of the Feynman diagrams in Figs. 3-6 can be computed by using $D_{\mu \nu}^{a b}(p)$ in eq. (2.6) as the effective gauge field propagator. By taking into account that the IR dimension of $D_{\mu \nu}^{a b}(p)$ is -1 , one may readily see that every single diagram in Figs. 3-6 is IR convergent by power counting at $p \neq 0$. Thus, no IR singularities occur as $D \rightarrow 3$. In accordance with [45], one-loop subintegrations in the diagrams at hand do not give rise to UV singularities as $D \rightarrow 3$ either, although they might correspond to superficially UV divergent one-loop diagrams. However, overall UV singularities do 
appear at two loops in individual Feynman diagrams as $D$ approaches 3 . In fact, the two-loop dimensionally regularized diagrams of Figs. 4, 5 and 6 will develop UV poles at $x=D-[r / 2]$ as $D \rightarrow 3$ if $x$ is zero or a positive integer, where $r$ is the UV degree of the integrand and $[r / 2]$ is the largest integer less or equal than $r / 2$. For the diagrams we are considering $r$ is equal to 6 so that $x=0$. Notice that the diagram in Fig. 3 does not exhibit UV divergences, since it factores into the product of two one-loop diagrams.

Let us now discuss the tensor structure of these overall UV singularities. The UV degree of the term in $D_{\mu \nu}^{a b}(p)$ involving the antisymmetric quantity $\epsilon_{\mu \rho \nu}$ is one unit less than that of the whole $D_{\mu \nu}^{a b}(p)$. This very situation is met when studying the UV degree of the different terms in the three-gauge vertex $V_{\mu \rho \nu}^{a b c}(p, q, r)$. Then, if $\omega_{m}$ is the overall UV degree of a diagram at $D=3$, any integral with $M$ such antisymmetric objects arising from the diagram will have an overall UV degree equal to $\omega_{m}-M$. The fact that $\omega_{m}=0$ for the diagrams in Figs. 4,5 and 6 [see eq. (2.13)] implies that the only integrals exhibiting singularities at $D=3$ are what we might call "pure" Yang-Mills integrals, i.e. those obtained by formally setting to zero every $\epsilon_{\mu \rho \nu}$ in the diagram. One may show as well that any term of these two-loop pure Yang-Mills integrals with at least one external momentum in the numerator has a negative overall UV degree. Thus, the only sources of UV singularities at $D=3$ are two-loop integrals having neither $\epsilon_{\mu \rho \nu}$ nor external momenta in their numerators. The formal version of these integrals at $D=n$ is $S O(n)$ invariant and we have seen that they have logarithmic superficial degree of divergence at $D=3$. Moreover, they are IR finite at $p \neq 0$ and without UV subdivergences as $D \rightarrow 3$. Hence, their singularity is a simple pole at $D=3$ independent of $m$ and the external momentum $p[43,45]$. In addition, they are always multyplied by $m$ since the mass dimension of the vacuum polarization tensor is one. We then conclude that the singular contribution coming from the diagrams in Figs. 4, 5 and 6 has tensor structure

$$
c \frac{m}{D-3} g_{\mu \nu}
$$


where $c$ is a real number whose value depends on the diagram.

We shall show in this section, by explicit computation, that the UV singularities (5.2) cancel when one sums over all two-loop diagrams contributing to $\Pi_{\mu \nu}^{a b}(p, m, D)$. Let us remind that from the point of view of the naïve dimensional regularization of Subsect. 2.1, this was a necessary step to recover BRS invariance in the 3-dimensional limit. On the contrary, from the point of view of the BRS-invariant dimensional regularization defined in Subsect. 2.2, finiteness or cancelation of singularities is a consequence of BRS invariance at the regularized level. Indeed, eq. (2.32) and the fact that the singular part of $\Pi_{\mu \nu}^{(2.1)}$ as $D$ goes to 3 is of the form (5.2) imply that the divergent part of $\Pi_{\mu \nu}^{(2.2)}$ as $D \rightarrow 3$ is of the form

$$
m\left(b_{1} \tilde{g}_{\mu \nu}+b_{2} \hat{g}_{\mu \nu}\right)
$$

with

$$
b_{1}=\frac{c}{D-3}-\operatorname{div}\left\{a_{1}\right\} \text { and } b_{2}=\frac{c}{D-3}-\operatorname{div}\left\{a_{2}\right\}
$$

and where $\operatorname{div}\{\cdots\}$ denotes the singular part in the MS sheme as $D$ approaches 3. Since the dimensionally regularized theory is BRS-invaraint, $\Pi_{\mu \nu}^{(2.2)}$ is transverse with respect to the external momentum thus having $b_{1}=b_{2}=0$.

We next come to explicit computations. There are sixteen two-loop diagrams that contribute to the polarization tensor of the gauge field, see Figs. 3-6. We already know that poles come from overall logarithmic UV divergences. A way to check the finiteness of the vacuum polarization tensor for (3-dimensional) TMYM theory is to collect all overall divergent integrals from all diagrams, compute them, and sum the corresponding results. Everything in this process, but for the evaluation of the integrals, is a question of algebra and can be performed with the help of an algebraic language, REDUCE in our case. Here we show with an example how to compute the integrals and present the final result. 
Let us consider the diagrams in Fig. 4. A typical superficially UV divergent contribution is

$$
m g^{4} c_{V}^{2} \delta^{a b} \int \frac{d^{D} k}{(2 \pi)^{D}} \frac{d^{D} q}{(2 \pi)^{D}} \frac{k^{2} q^{8}(k q) q_{\mu} q_{\nu}}{D_{4}(k, q, p, m)}
$$

where the denominator in the integrand is given by

$$
\begin{aligned}
D_{4}(k, q, p, m) & =k^{2}\left(k^{2}+m^{2}\right)(k+q)^{2}\left[(k+q)^{2}+m^{2}\right] \\
& \times q^{4}\left(q^{2}+m^{2}\right)^{2}(q+p)^{2}\left[(q+p)^{2}+m^{2}\right]
\end{aligned}
$$

Note that the integral (5.3) has overall UV logarithmic degree of divergence, in accordance with what has been said above. To eliminate the dependence on the external momentum $p$ of the divergences we use the algebraic identities

$$
\begin{aligned}
\frac{1}{(q+p)^{2}} & =\frac{1}{q^{2}}-\frac{2 p q+p^{2}}{q^{2}(q+p)^{2}} \\
\frac{1}{\left[(q+p)^{2}+m^{2}\right]} & =\frac{1}{q^{2}+m^{2}}-\frac{2 p q+p^{2}}{\left(q^{2}+m^{2}\right)\left[(q+p)^{2}+m^{2}\right]} .
\end{aligned}
$$

The integrals arising from the $p$-dependent terms on the RHS of these identities are overall UV convergent at $D=3$. Moreover, by inspection we see that they are also IR finite at $p=0$. The $o$-theorem then implies that they vanish in the limt $m \rightarrow \infty$, since the inequality (3.5) now takes the form $\left[n_{k}+n_{q}\right]+n_{p}>1$, with $n_{k}, n_{q}$ and $n_{p}$ the number of $k, q$ and $p$ in the numerator of the corresponding integrands. Thus, poles are independent of $p$ and to compute them we can take $p=0$ in $D_{4}$ so we can rewrite $(5.3)$ as

$$
m g^{4} c_{V}^{2} \delta^{a b} \int \frac{d^{D} k}{(2 \pi)^{D}} \frac{d^{D} q}{(2 \pi)^{D}} \frac{k^{2} q^{8}(k q) q_{\mu} q_{\nu}}{D_{4}(k, q, 0, m)}+\text { v.t. }
$$

where "v.t." stands for finite contributions at $D=3$ which vanish as $m$ goes to $\infty$. Rescaling the integration variables $k \rightarrow k m, q \rightarrow q m$ and using $S O(D)$ 
invariance, we have:

$$
\frac{m}{D} g^{4} c_{V}^{2} \delta^{a b} g_{\mu \nu} \int \frac{d^{D} k}{(2 \pi)^{D}} \frac{d^{D} q}{(2 \pi)^{D}} \frac{k^{2} q^{10}(k q)}{D_{4}(k, q, 0,1)}+\text { v.t. }
$$

where now the numerator in the integrand is a scalar. The next step is to use algebraic identities of the type

$$
\begin{aligned}
& \frac{2 k q}{(k+q)^{2}}=1-\frac{q^{2}}{(k+q)^{2}}-\frac{k^{2}}{(k+q)^{2}}, \\
& \frac{k^{2}}{k^{2}+1}=1-\frac{1}{k^{2}+1}, \\
& \frac{1}{k^{2}\left(k^{2}+1\right)}=\frac{1}{k^{2}}-\frac{1}{k^{2}+1}
\end{aligned}
$$

to decompose (5.6) into simpler integrals. The Appendix contains a list of all the integrals found at the end of this simplification process for the diagrams in Figs. 4-6, as well as their values. Using the results presented there we get for the integral in $(5.6)$ :

$$
\frac{1}{64 \pi^{2}}\left[-\frac{1}{\varepsilon}+1-\gamma+\frac{325}{72}+\ln \left(\frac{729 \pi}{64}\right)+O(\varepsilon)\right],
$$

where $\gamma$ is Euler-Mascheroni's constant and $\varepsilon=D-3$. Notice that (5.8) exhibits a simple pole at $D=3$. UV divergent integrals from the diagrams in Figs. 5 and 6 are studied in the same way. For them we always obtain the same structure as for (5.6), namely a scalar divergent integral times $m g_{\mu \nu}$. Regarding the diagram of Fig. 3, it factores into the product of two one-loop diagrams and therefore is free of poles. After summing over all diagrams we obtain

$$
\Pi_{\mu \nu}^{(2) \mathrm{YM}}(p, m)=-m g_{\mu \nu}\left(\frac{1135}{864}+\frac{9}{4} \ln 2-\frac{13}{16} \ln 3\right)\left(\frac{g^{2} c_{V}}{4 \pi}\right)^{2}+\text { v.t. }
$$

which does not have any singularity. This proves that the vacuum polarization tensor for TMYM theory is finite at second order in perturbation theory. The superindex YM in eq. (5.9) is to remind that this is only the contribution from 
pure Yang-Mills terms, or equivalently, terms which by power counting are overall superficially UV divergent. For simplicity in the notation we have dropped colour indices.

Having shown that the (3-dimensional) topologically massive vacuum polarization tensor $\Pi_{\mu \nu}^{(2)}(p, m)$ is well-defined, our next goal is to compute its large $m$ limit. Let us remind that $\Pi_{\mu \nu}^{(2)}(p, m)$ is obtained by taking the limit $D \rightarrow 3$ of the sum of the diagrams in Figs. 3-6. The contribution from pure Yang-Mills integrals is given in eq. (5.9). We still have to calculate the finite contribution $\Pi_{\mu \nu}^{(2) \mathrm{F}}(p, m)$ coming from integrals involving one or more $\epsilon_{\mu \rho \nu}$. The naïve way to evaluate them would be to perform the whole computation keeping $m$ finite and then take the limit $m \rightarrow \infty$. From the point of view of the algebra involved, this presents the same degree of complexity as the computation of the gluon polarization tensor in QCD. The situation is much worse from the point of view of integration, since now there are massive denominators. Although this procedure is still viable at one loop [5], it must be given up if we intend to pursue our computation to higher orders in perturbation theory. We will use instead the vanishing theorems of Sect. 3.

We begin by applying these theorems to disregard integrals that go to zero as $m$ approaches infinity. To show how to do this we use once more the diagrams in Fig. 4 as an example. The most general integral arising from them is of the form

$$
I(p, m)=\int \frac{d^{D} k}{(2 \pi)^{D}} \frac{d^{D} q}{(2 \pi)^{D}} \frac{m^{n_{m}} p^{n_{p}} k^{n_{k}} q^{n_{q}}}{D_{4}(k, q, p, m)}
$$

where the denominator is given by (5.4). The $m$-theorem demands the following conditions to be met for the integral to go to zero as $m \rightarrow \infty$ :

$$
\begin{aligned}
& \text { Overall UV convergence : } n_{k}+n_{q}-14<0 \text {, } \\
& \text { IR convergence at } p \neq 0: n_{k}+n_{q}-2>0, n_{k}+1>0, n_{q}-1>0 \text {, } \\
& \text { Condition (3.3) : } n_{k}+n_{q}+n_{m}-14<0, n_{m}-10<0 \text {, } \\
& n_{k}+n_{m}-11<0, n_{q}+n_{m}-15<0 \text {, }
\end{aligned}
$$

where we have have already used that UV convergence along one-loop subintegrals 
can be dropped, as discussed at the end of Sect. 3. The Feynman rules as applied to the diagrams we are considering imply that the only non-trivially satisfied conditions are

$$
n_{k}+n_{q}+n_{m}-14<0 \text { and } n_{k}+n_{m}-11<0 .
$$

Analogously, one can obtain that the o-theorems demands:

$n_{k}+n_{q}-14<0, n_{k}+n_{q}-4>0, n_{q}-3>0,\left[n_{k}+n_{q}\right]+14-n_{k}-n_{q}-n_{m}>0$.

We now use the cuts (5.11) and (5.12) to keep only those integrals (5.10) which do not vanish in the limit $m \rightarrow \infty$. In this way we are left with a number of integrals that have to be computed. In what follows we give two examples of such integrals and show how to evaluate them. Again the $m$ - and $o$-theorems will play a very important part.

Let us first consider the integral

$$
I_{\mu \nu}(p)=\int \frac{d^{D} k}{(2 \pi)^{D}} \frac{d^{D} q}{(2 \pi)^{D}} \frac{m^{7} k^{4} q^{2} q_{\mu} q_{\nu}}{D_{4}(k, q, p, m)},
$$

with the denominator in the integrand given by (5.4). Note that $n_{k}+n_{q}+n_{m}=15$ and $\left[n_{k}+n_{q}\right]+14-n_{k}-n_{q}-n_{m}=0$ so that the theorems did not apply. This integral is IR finite at $p=0$ for $D=3$ so we must expect a contribution from $p=0$, which will be of order $m$, plus subleading contributions of order $m^{0}$. Using the identities (5.5) and the $o$-theorem it is not difficult to see that

$$
I_{\mu \nu}(p)=I_{\mu \nu}(0)+I_{\mu \nu}^{\prime}(p)+\text { v.t. },
$$

where

$$
I_{\mu \nu}^{\prime}(p)=-\int \frac{d^{D} k}{(2 \pi)^{D}} \frac{d^{D} q}{(2 \pi)^{D}} \frac{m^{7} k^{4} q^{2}\left(2 p q+p^{2}\right) q_{\mu} q_{\nu}}{D_{4}^{\prime}(k, q, p, m)}
$$


and the denominator $D_{4}^{\prime}$ reads

$$
D_{4}^{\prime}(k, q, p, m)=k^{2}\left(k^{2}+m^{2}\right)(k+q)^{2}\left[(k+q)^{2}+m^{2}\right](p+q)^{2} q^{6}\left(q^{2}+m^{2}\right)^{3} .
$$

The integral $I_{\mu \nu}(0)$ is of the same type as those that appeared when studying the pure Yang-Mills sector, with the difference that this one is finite at $D=3$. It can be computed in the same way, the result being

$$
I_{\mu \nu}(0)=m g_{\mu \nu} \frac{1}{48 \pi^{2}}\left(\frac{143}{288}-2 \ln 2+\ln 3\right) .
$$

Regarding $I_{\mu \nu}^{\prime}(p)$, it can be further simplified. To decouple integration over $k$ from that over $q$ we use the algebraic identities

$$
\begin{aligned}
\frac{1}{(k+q)^{2}} & =\frac{1}{k^{2}}-\frac{2 k q+q^{2}}{k^{2}(k+q)^{2}}, \\
\frac{1}{(k+q)^{2}+m^{2}} & =\frac{1}{k^{2}+m^{2}}-\frac{2 k q+q^{2}}{\left(k^{2}+m^{2}\right)\left[(k+q)^{2}+m^{2}\right]} .
\end{aligned}
$$

The terms with dependence on $q$ on the RHS of (5.14) give zero contribution to $I_{\mu \nu}^{\prime}(p)$ when $m \rightarrow \infty$ by application of the $o$-theorem. We thus get for $I_{\mu \nu}^{\prime}(p)$ the product of two one-loop integrals:

$$
I_{\mu \nu}^{\prime}(p)=-\int \frac{d^{D} k}{(2 \pi)^{D}} \frac{m}{\left(k^{2}+m^{2}\right)^{2}} \int \frac{d^{D} q}{(2 \pi)^{D}} \frac{m^{6} q^{2}\left(2 p q+p^{2}\right) q_{\mu} q_{\nu}}{(q+p)^{2} q^{6}\left(q^{2}+m^{2}\right)^{3}}+\text { v.t. . }
$$

Rescaling $k \rightarrow k m$ we concentrate all the dependence on $m$ on the integral over $q$. Using then identities of the type (5.7) and the o-theorem we finally get that

$$
\begin{aligned}
I_{\mu \nu}^{\prime}(p) & =\int \frac{d^{D} k}{(2 \pi)^{D}} \frac{1}{\left(k^{2}+1\right)^{2}} \int \frac{d^{D} q}{(2 \pi)^{D}} \frac{q_{\mu} q_{\nu}}{q^{2}(q+p)^{2}}+\text { v.t. } \\
& =-\frac{1}{512 \pi \sqrt{p^{2}}}\left(p^{2} g_{\mu \nu}-3 p_{\mu} p_{\nu}\right)+\text { v.t. },
\end{aligned}
$$

which is of order $m^{0}$. Another typical example of an integral with non-zero limit 
$m \rightarrow \infty$ is

$$
\int \frac{d^{D} k}{(2 \pi)^{D}} \frac{d^{D} q}{(2 \pi)^{D}} \frac{m^{9} k^{2} q^{2} p_{\mu} q_{\nu}}{D_{4}(k, q, p, m)}=-\frac{p_{\mu} p_{\nu}}{128 \pi \sqrt{p^{2}}}+\text { v.t. . }
$$

In this case the integral had $n_{k}+n_{q}+n_{m}=14$ and was IR divergent at $p=0$ for $D=3$ so the theorems did not apply. Nevertheless, its large $m$ limit has a simple expression. Note that both examples yield non-local terms.

All the integrals from the diagrams in Figs. 3-6 that do not vanish when $m$ goes to $\infty$ can be computed in the same way as these two examples. When we sum over diagrams, non-localities cancel and the contribution to the polarization tensor from terms with one or more $\epsilon_{\mu \nu \rho}$ takes the form:

$$
\begin{aligned}
\Pi_{\mu \nu}^{(2) \mathrm{F}} & =m g_{\mu \nu}\left(\frac{1135}{864}+\frac{9}{4} \ln 2-\frac{13}{16} \ln 3\right)\left(\frac{g^{2} c_{V}}{4 \pi}\right)^{2} \\
& +\epsilon_{\mu \rho \nu} p^{\rho}\left(\frac{265}{36}+\frac{44}{3} \ln 2-\frac{63}{4} \ln 3\right)\left(\frac{g^{2} c_{V}}{4 \pi}\right)^{2}+\text { v.t. . }
\end{aligned}
$$

Combining this result with the contribution (5.9), we finally get

$$
\Pi_{\mu \nu}^{(2) a b}(p, m)=\epsilon_{\mu \rho \nu} p^{\rho} \delta^{a b} \frac{265+L}{36}\left(\frac{g^{2} c_{V}}{4 \pi}\right)^{2}
$$

where we have reinserted colour indices and introduced the constant

$$
L=528 \ln 2-567 \ln 3 .
$$

We thus see that when the limit $m \rightarrow \infty$ is taken no infinities appear. Eq. (5.16) gives the $g^{4}$-correction to the CS gauge field polarization tensor and will be used in Sect. 8 to construct the effective action.

Notice that since limit $m \rightarrow \infty$ prserves BRS invariance, the regularized CS vacuum polarization tensor must be transverse. Hence, if the $m g_{\mu \nu}$ terms 
in $\Pi_{\mu \nu}^{(2) \mathrm{YM}}$ in eq. (5.9) and $\Pi_{\mu \nu}^{(2) \mathrm{F}}$ in eq. (5.15) did not cancel one another, transversality would be lost. Finally, let us point out that the absence of non-local large $m$ divergent terms is due to the lack of UV subdivergences after summing over all two-loop diagrams, or in other words, to UV finiteness of one-loop 1PI functions as $m \rightarrow \infty$.

\section{The ghost self-energy and the ghost-ghost-external field vertex}

One of our aims is to construct the local part of the two-loop effective action for CS theory. We will do this in the next section. To determine the coefficients of the terms entering in the effective action we will need to know three Green functions. We have already taken one of them to be the gauge field propagator, which was studied in Sect. 5. For the other two we chose the ghost propagator and the ghost-ghost-external field vertex, whose results we give here.

Let us first consider the ghost propagator. We want to compute the ghost selfenergy $\Omega^{a b}(p)$ up to second order in perturbation theory. At the first perturbative order there is only one diagram that contributes to the self-energy, see Fig. 7. Its contribution in the limit $m \rightarrow \infty$ can be easily computed [5,22], the result being

$$
\Omega^{(1) a b}(p)=-\frac{2}{3} \frac{g^{2} c_{V}}{4 \pi} p^{2} \delta^{a b} .
$$

At second order there are six diagrams that contribute, see Fig. 8. We use the theorems of Sect. 3 to keep only those integrals that do not vanish as $m$ goes to $\infty$. Their evaluation in the limit $m \rightarrow \infty$ goes along the lines explained in the last section. As a matter of fact, it happens that all the integrals one has to evaluate are among those studied in Sect. 5. Here we limit ourselves to give the result:

$$
\Omega^{(2) a b}(p)=-\frac{169+L}{72}\left(\frac{g^{2} c_{V}}{4 \pi}\right)^{2} p^{2} \delta^{a b}
$$

where $L$ is as in eq. (5.17). 
It is worth noticing that despite power counting as applied to CS theory predicts linear UV divergences, the results in (6.1) and (6.2) are finite, in agreement with $[27,28]$. Moreover, first and second order corrections to the ghost self-energy in TMYM theory are also finite: they are given in terms of finite integrals whose large $m$ limit precisely gives (6.1) and (6.2). Again, this is in contrast with the logarithmic UV divergences predicted by power counting for TMYM one-loop diagrams.

We next study the ghost-ghost-external field vertex $H^{a} c^{b} c^{c}$. At one loop there is only one Feynman diagram (see Fig. 9). It is not difficult to shwow that its contribution to the vertex vanishes in the $m \rightarrow \infty$ limit,

$$
V^{(1) a b c}\left(p_{1}, p_{2}\right)=0+\text { v.t. }
$$

As for second order corrections, Fig. 10 depicts all two-loop diagrams that contribute. The way to compute them is analogous to that of calculating the ghost self-energy and gauge field vacuum polarization tensor. The only difference is that now it is enough to use the $m$-theorem. Let us illustrate the method for the diagram in Fig 10(a). We get for its contribution:

$$
\frac{1}{4} g^{5} c_{V}^{2} f^{a b c} \int \frac{d^{3} k}{(2 \pi)^{3}} \frac{d^{3} q}{(2 \pi)^{3}} \frac{N\left(k, q, p_{1}, p_{2}, m\right)}{D_{10}\left(k, q, p_{1}, p_{2}, m\right)},
$$

where the numerator and denominator of the integrand are given by

$$
\begin{aligned}
N\left(k, q, p_{1}, p_{2}, m\right) & =m^{2} p_{1}^{\mu} p_{2}^{\nu} q^{\rho}\left(q+p_{1}+p_{2}\right)^{\sigma} \\
& \times\left[m \epsilon_{\mu \tau \nu}\left(q+p_{1}\right)^{\tau}+\left(q+p_{1}\right)^{2} \delta_{\mu \nu}-\left(q+p_{1}\right)_{\mu}\left(q+p_{1}\right)_{\nu}\right] \\
& \times\left[m \epsilon_{\rho \lambda \sigma} k^{\lambda}+k^{2} \delta_{\rho \sigma}-k_{\rho} k_{\sigma}\right]
\end{aligned}
$$

and

$$
\begin{aligned}
D_{10}\left(k, q, p_{1}, p_{2}, m\right) & =q^{2}\left(q+p_{1}\right)^{2}\left[\left(q+p_{1}\right)^{2}+m^{2}\right]\left(q+p_{1}+p_{2}\right)^{2} \\
& \times k^{2}\left(k^{2}+m^{2}\right)(k+q)^{2}\left(k+q+p_{1}+p_{2}\right)^{2}
\end{aligned}
$$

For non-zero external momenta, the diagram is by power counting finite. The $m$-theorem then demands that for (6.4) to vanish as $m \rightarrow \infty$, condition (3.3) 
must hold. But condition (3.3) requires the following inequalities to be satisfied simultaneously:

$$
n_{k}+n_{q}+n_{m}-10<0, n_{k}+n_{m}-7<0, n_{q}+m_{m}-11<0, n_{m}-4<0,
$$

where as usual $n_{k}, n_{q}$ and $n_{m}$ denote the number of $k^{\prime} \mathrm{s}, q^{\prime} \mathrm{s}$ and $m^{\prime} \mathrm{s}$ in the numerator of the integrand. The only term that violates these requirements is

$$
m^{4} p_{1}^{\mu} p_{2}^{\nu} q^{\rho}\left(p_{1}+p_{2}\right)^{\sigma} \epsilon_{\mu \tau \nu} q^{\tau} \epsilon_{\rho \lambda \sigma} k^{\lambda}
$$

for which $n_{m}=4$. Now, integration over $k$ yields a linear combination of $q^{\lambda}$ and $\left(p_{1}+p_{2}\right)^{\lambda}$ that together with the $\epsilon_{\rho \lambda \sigma} q^{\rho}\left(p_{1}+p_{2}\right)^{\sigma}$ gives zero. Proceeding in a similar way we obtain for the other diagrams the following. The colour algebra of the diagram in Fig. 10.(b) is already zero. The $m$-theorem implies that each one of the diagrams in Figs. 10(c)-10(e) on its own vanishes as $m$ goes to $\infty$. Finally, the diagrams in Figs. 10(f) and 10(g) on the one hand, and Figs. 10(h) and 10(i) on the other, combine to give zero contribution for $m \rightarrow \infty$. We thus obtain that

$$
V^{(2) a b c}\left(p_{1}, p_{2}\right)=0
$$

This result and (6.2) will be used in the Sect. 8 .

\section{Perturbative finiteness of TMYM theory}

Here we collect some results from previous sections that put together imply perturbative finiteness for TMYM theory. It follows from eq. (2.13) that the only 1PI Green functions that are not finite by power counting are:

a) The gauge field one-point function $\left\langle A_{\mu}^{a}\right\rangle$, with eq. (2.13) predicting quadratic, linear and logarithmic UV divergences at one, two and three loops respectively. 
b) The vacuum polarization tensor, for which power counting gives linear UV divergences at one loop and logarithmic at two.

c) The ghost self-energy and the vertex $\left\langle A_{\mu}^{a} A_{\nu}^{b} A_{\rho}^{c}\right\rangle$, power counting yielding one-loop logarithmic UV divergences.

To give a well-defined meaning to these Green functions we have introduced dimensional regularization in Sect. 2.

As shown in Sect. 4, any 1PI diagram contributing to $\left\langle A_{\mu}^{a}\right\rangle$ is zero by colour algebra upon regularization. This leaves us with only b) and c) above as sources of UV divergences. In Sect. 2 we have seen that the limit $D \rightarrow 3$ of every dimensionally regularized one-loop 1PI Feynman diagram is free of singularties, despite power counting might predict divergences. This ensures finiteness at one loop. Furthermore, being the limit $D \rightarrow 3$ finite at one loop, it can be regarded as defining a renormalization scheme at this order. Thus, two-loop UV divergences may only come from the vacuum polarization tensor. In Sect. 5 we have seen that although individual two-loop 1PI diagrams contributing to the polarization tensor have poles at $D=3$, the latter cancel when summing over diagrams thus providing a finite polarization tensor. Hence, no UV divergences arise at second order in perturbation theory. Again, the limit $D \rightarrow 3$ defines a renormalization scheme at this order. This, combined with finiteness by power counting at higher loops implies that TMYM theory is finite at any perturbative order.

Finiteness at one loop was proved in [3,5]. At higher loops, though expected, has remained unproved. Also in [3,5] explicit expressions for one-loop radiative corrections can be found. Finding compact expressions for such corrections beyond one loop seems today a task beyond human (and computer) capability. In this paper we have computed the large $m$ limit of some 1PI functions at two loops 


\section{The bare effective action}

In this section we compute the first and second order radiative corrections to the bare CS effective action $\Gamma$ up to order three in the fields. We shall work in the Landau gauge, as we have done so far. The bare effective action is defined to be

$$
\Gamma=\Gamma(A, c, \bar{c}, b, J, H ; k)=\lim _{m \rightarrow \infty} \lim _{D \rightarrow 3} \Gamma(A, c, \bar{c}, b, J, H ; k ; m, D)
$$

wherever the previous sequence of limits exists. We will assume for the time being that the above double limit is indeed finite up to second order in perturbation theory and postpone its proof until the end of this section. This amounts to assuming UV finiteness of $\Gamma$ up to two loops. Note that this is in agreement with the general finiteness proofs in [27] and [28]. In (8.1), the integrated functional $\Gamma(A, c, \bar{c}, b, J, H ; k ; m, D)$ is the generating functional of the amputated 1PI functions regularized through any of the regularization methods in Sect. 2. Let us recall that both methods yield the same result in the limit $D \rightarrow 3$.

Throughout this section we will write $\Gamma$ as a function of $k>0$, the classical or bare CS parameter, rather than as a function of the classical or bare coupling constant $g=(4 \pi / k)^{1 / 2}$. This can be accomplished by introducing the following scalings of the fields:

$A^{\mu a} \rightarrow \frac{1}{g} A^{\mu a} \quad, \quad b^{a} \rightarrow g b^{a} \quad, \quad c^{a} \rightarrow \frac{1}{g} c^{a} \quad, \quad \bar{c}^{a} \rightarrow g \bar{c}^{a} \quad, \quad J^{\mu a} \rightarrow g J^{\mu a} \quad, \quad H^{a} \rightarrow g H^{a}$

In computing $\Gamma$ we will use the BRS identities, the values of the 1PI functions calculated in previous sections and the following result. Both the one-loop and the two-loop terms in the effective action are local integrated functionals of the fields, provided they correspond to 1PI functions with less than than four fields. This statement is a consequence of two facts: $(i)$ the regularization method in [18] yields vanishing radiative corrections up to two loops for the 1PI functions here considered, and ( $i i)$ two renormalization prescriptions for the effective action that agree at $n$ loops can only differ by a local functional at $n+1$ loops[52]. 
As for the BRS identities, we are going to write them in terms of the functional $\bar{\Gamma}:$

$$
\bar{\Gamma}=\Gamma+\int d^{3} x b^{a} \partial A^{a}
$$

with $\Gamma$ as in eq. (8.1). The functional $\bar{\Gamma}$ satisfies the following equations:

$$
\begin{gathered}
\frac{\delta \bar{\Gamma}}{\delta b^{a}}=0, \quad\left(\partial_{\mu} \frac{\delta}{\delta J_{\mu}^{a}}-\frac{\delta}{\delta \bar{c}^{a}}\right) \bar{\Gamma}=0 \\
\int d^{3} x\left(\frac{\delta \bar{\Gamma}}{\delta A^{\mu a}} \frac{\delta \bar{\Gamma}}{\delta J_{\mu}^{a}}+\frac{\delta \bar{\Gamma}}{\delta c^{a}} \frac{\delta \bar{\Gamma}}{\delta H^{a}}\right)=0 .
\end{gathered}
$$

Let us discuss why eqs. (8.3) and (8.4) hold. The two equations in (8.3) are the Landau gauge-fixing condition and the antighost equation of motion respectively. They hold for the regularized $\bar{\Gamma}(A, c, \bar{c}, b, J, H ; k ; m, D)$ obtained through any of the regularization methods in Sect. 2 and the $D$-dimensional analogue of eq. (8.2). Eq. (8.4) is the BRS equation. This equation is satisfied by the regularized effective action $\bar{\Gamma}(m, D)$ that the second regularization method in Sect. 2 provides (see Subsect. 2.2). Let us recall that this method is explicitly BRS-invariant and that eq. (8.4) is the equation ruling BRS invariance for the quantum theory. On the other hand, the first regularization method in Sect. 2 (see Subsect. 2.1) yields a regularized effective action $\bar{\Gamma}(m, D)$ that does not satisfy the BRS equation. However, we have shown that the terms breaking the BRS symmetry go to zero as $D \rightarrow 3$. Moreover, both regularization methods yield the same effective action in the limit $D \rightarrow 3$. We then conclude that $\bar{\Gamma}$ in eq. (8.2) does satisfy the set of equations above, hence that it is BRS-invariant.

The first equation (8.3) implies that $\bar{\Gamma}$ does not depend on $b^{a}$. In turn, the antighost equation leads to the conclusion that $J_{\mu}^{a}$ and $\bar{c}^{a}$ always occur through the combination

$$
G_{\mu}^{a}(x)=J_{\mu}^{a}(x)-\partial_{\mu} \bar{c}^{a}(x)
$$

Thus, the functional $\bar{\Gamma}$ should be understood as a function of $A_{\mu}^{a}, G_{\mu}^{a}, c^{a}$ and $H^{a}$. As an integrated functional, $\bar{\Gamma}$ has mass dimension three and ghost number 
zero. The fields $A_{\mu}^{a}, G_{\mu}^{a}, c^{a}$ and $H^{a}$ all have mass dimesion 1 and ghost number $1,-1,1$ and -2 respectively. From these mass dimensions and ghost numbers it follows that contributions to $\bar{\Gamma}$ quadratic or cubic in the fields are local, whereas contributions quartic or higher are purely non-local functionals, i.e. with no local part. Recall CS theory in the Landau gauge does not involve any dimensionful parameter.

As for eq. (8.4), it establishes relations among the coefficients of the 1PI functions contributing to $\bar{\Gamma}$. These relations, toghether with the values of the 1PI functions we have computed in previous sections, fix completely the local part of $\bar{\Gamma}(A, c, G, H ; k)$ up to two loops. To see this we introduce a loop-wise expansion for $\bar{\Gamma}$ :

$$
\bar{\Gamma}=\sum_{n=0}^{\infty} \hbar^{n} \bar{\Gamma}^{(n)}
$$

where $\bar{\Gamma}^{(0)}$ is the tree-level effective action minus the gauge-fixing term, or more precisely:

$$
\bar{\Gamma}^{(0)}=S_{C S}+\int d^{3} x\left(G^{a \mu} D_{\mu}^{a b} c^{b}-\frac{1}{2} f^{a b c} H^{a} c^{b} c^{c}\right) .
$$

The symbol $S_{C S}$ stands for the CS classical action in terms of $k$,

$$
S_{C S}=-\frac{i k}{4 \pi} \int d^{3} x \epsilon^{\mu \rho \nu}\left(\frac{1}{2} A_{\mu}^{a} \partial_{\rho} A_{\nu}^{a}+\frac{1}{3 !} f^{a b c} A_{\mu}^{a} A_{\rho}^{b} A_{\nu}^{c}\right) .
$$

Substitution of eq. (8.6) in eq. (8.4) gives the following equations for the one-loop $\bar{\Gamma}^{(1)}$ and two-loop $\bar{\Gamma}^{(2)}$ contributions to $\bar{\Gamma}$ :

$$
\Delta \bar{\Gamma}^{(1)}=0,
$$

and

$$
\int d^{3} x\left[\frac{\delta \bar{\Gamma}^{(1)}}{\delta A^{\mu a}} \frac{\delta \bar{\Gamma}^{(1)}}{\delta G_{\mu}^{a}}+\frac{\delta \bar{\Gamma}^{(1)}}{\delta c^{a}} \frac{\delta \bar{\Gamma}^{(1)}}{\delta H^{a}}\right]+\Delta \bar{\Gamma}^{(2)}=0 .
$$


Here $\Delta$ is the Slavnov-Taylor operator,

$$
\Delta=\int d^{3} x\left[\frac{\delta \bar{\Gamma}^{(0)}}{\delta A^{\mu a}} \frac{\delta}{\delta G_{\mu}^{a}}+\frac{\delta \bar{\Gamma}^{(0)}}{\delta G_{\mu}^{a}} \frac{\delta}{\delta A^{\mu a}}+\frac{\delta \bar{\Gamma}^{(0)}}{\delta c^{a}} \frac{\delta}{\delta H^{a}}+\frac{\delta \bar{\Gamma}^{(0)}}{\delta H^{a}} \frac{\delta}{\delta c^{a}}\right]
$$

As is well-known, $\Delta$ is nilpotent, $\Delta^{2}=0$. Notice that in passing from eq. (8.4) to eqs. (8.8) and (8.9) we have taken into account that

$$
\frac{\delta \bar{\Gamma}}{\delta J_{\mu}^{a}}=\frac{\delta \bar{\Gamma}}{\delta G_{\mu}^{a}} .
$$

Eq. (8.8) imposes some constraints upon the local part $\bar{\Gamma}_{\text {local }}^{(1)}$ of $\bar{\Gamma}^{(1)}$. The most general way to find them would be to solve the equation

$$
\Delta W=0
$$

over the space of integrated functionals $W$ of mass dimension three and ghost number zero that depend on $A_{\mu}^{a}, c^{a}, G_{\mu}^{a}$ and $H^{a}$. In principle, $W$ has local and non-local contributions and, in perturbation theory, can be expressed as the sum

$$
W(A, G, c, H)=\sum_{i=2}^{\infty} W_{i}(A, G, c, H)
$$

with the index $i$ counting the number of fields in $W_{i}$. Note that we have restricted the sum to $i \geq 2$, since contributions to the effective action $\bar{\Gamma}$ at any order in perturbation theory, and in particular to $\bar{\Gamma}^{(1)}$, are at least quadratic in the fields, as shown in Sect. 4. Furthermore, as we have already mentioned, contributions of order two and three in the fields are local, and contributions of order four or higher are non-local. This means that $W_{2}$ and $W_{3}$ are local functionals and that $W_{i}(i \geq 4)$ is purely non-local. Regarding the Slavnov-Taylor operator $\Delta$, it is convenient to split it also into two terms, each one of them of a given order in the 
number of fields. To do this we decompose $\bar{\Gamma}^{(0)}$ in the sum of its quadratic $\bar{\Gamma}_{2}^{(0)}$ and cubic $\bar{\Gamma}_{3}^{(0)}$ parts in the fields,

$$
\bar{\Gamma}^{(0)}(A, c, G, H)=\bar{\Gamma}_{2}^{(0)}(A, c, G)+\bar{\Gamma}_{3}^{(0)}(A, c, G, H) .
$$

Calling now $\Delta_{0}$ and $\Delta_{1}$ to the Slavnov-Taylor operators for $\bar{\Gamma}_{2}^{(0)}$ and $\bar{\Gamma}_{3}^{(0)}$ respectively, we have that

$$
\Delta=\Delta_{0}+\Delta_{1}
$$

with

$$
\Delta_{0}^{2}=\Delta_{1}^{2}=\left\{\Delta_{0}, \Delta_{1}\right\}=0
$$

We are interested in the most general $W_{2}$ and $W_{3}$ entering in the solution $W$ of eq. (8.11), since our $\bar{\Gamma}_{\text {local }}^{(1)}$ is the sum of two specific $W_{2}$ and $W_{3}$. Eq. (8.11) leads to an infinite number of coupled equations for the functionals $W_{i}$. However, only three of them involve $W_{2}$ and $W_{3}$, namely:

$$
\Delta_{0} W_{2}=0, \Delta_{0} W_{3}+\Delta_{1} W_{2}=0
$$

and

$$
\Delta_{1} W_{3}+\Delta_{0} W_{4}=0
$$

The last equation containing the purely non-local contribution $W_{4}$. It can be readily seen after some algebra that the most general integrated functional $W_{\text {local }}=$ $W_{2}+W_{3}$, with $W_{2}$ and $W_{3}$ solutions of eq. (8.15), is

$$
\begin{aligned}
W_{\text {local }}= & -\frac{i k}{4 \pi} \int d^{3} x \epsilon^{\mu \rho \nu}\left[\frac{1}{2}\left(w_{1}+2 w_{2}\right) A_{\mu}^{a} \partial_{\rho} A_{\nu}^{a}+\frac{1}{3 !}\left(w_{1}+3 w_{2}\right) f^{a b c} A_{\mu}^{a} A_{\rho}^{b} A_{\nu}^{c}\right] \\
& +\int d^{3} x\left[-w_{2} G_{\mu}^{a} \partial^{\mu} c^{a}+w_{3} G_{\mu}^{a}\left(D^{\mu} c\right)^{a}-\frac{w_{3}}{2} f^{a b c} H^{a} c^{b} c^{c}\right],
\end{aligned}
$$

where $w_{1}, w_{2}$ and $w_{3}$ are arbitrary coefficients. Actually, it is easy to check that the first equation in (8.15) holds for any functional $W_{2}$ of mass dimension three 
and ghost number zero. The functional $W_{\text {local }}$ can be recast into the form

$$
W_{\text {local }}(A, G, c, H)=w_{1} S_{C S}+\Delta X
$$

with

$$
X=\int d^{3} x\left(w_{2} G_{\mu}^{a} A^{a \mu}-w_{3} H^{a} c^{a}\right) .
$$

Now, it is well-known [27] that (8.18) is the most general solution of the equation

$$
\Delta W_{\text {local }}=0
$$

over the space of integrated local functionals of the fields $A_{\mu}^{a}, G_{\mu}^{a}, c^{a}$ and $H^{a}$ with mass dimension three and ghost number zero. From eqs. (8.20) and (8.14) it then follows that $\Delta_{1} W_{3}$ vanishes for any $W_{3}$ solving eq. (8.15). This, along with eq. (8.16), implies that $\Delta_{0} W_{4}=0$ so that the purely non-local sector of $W$, defined in eq. (8.12), decouples from the local sector as far as BRS invariance is concerned. This is also a property of $\bar{\Gamma}_{\text {local }}^{(1)}$, for it belongs to the space of functionals spanned by $W_{\text {local }}$.

To find the values of $w_{1}, w_{2}$ and $w_{3}$ that enter in $\bar{\Gamma}_{\text {local }}^{(1)}$ we exploit that the polarization tensor $\Pi_{\mu \nu}^{a b}(p)$, the ghost self-energy $\Omega^{a b}(p)$ and the external vertex $V^{a b c}\left(p_{1}, p_{2}\right)$ are generated by functional derivatives of $\bar{\Gamma}$ with respect to the fields:

$$
\begin{gathered}
\int \frac{d^{3} p}{(2 \pi)^{3}} \mathrm{e}^{-i p(x-y)} \Pi_{\mu \nu}^{a b}(p)=-\left.\frac{\delta^{2} \bar{\Gamma}}{\delta A^{\mu a}(x) \delta A^{\nu b}(y)}\right|_{A=0} \\
\int \frac{d^{3} p}{(2 \pi)^{3}} \mathrm{e}^{-i p(x-y)} \Omega^{a b}(p)=-\left.\frac{\delta^{2} \bar{\Gamma}}{\delta c^{a}(x) \delta \bar{c}^{b}(y)}\right|_{\bar{c}=c=0}, \\
\int \frac{d^{3} p_{1}}{(2 \pi)^{3}} \frac{d^{3} p_{2}}{(2 \pi)^{3}} \mathrm{e}^{-i p_{1}(x-z)} \mathrm{e}^{-i p_{2}(y-z)} V^{a b c}\left(p_{1}, p_{2}\right)=-\left.\frac{\delta^{3} \bar{\Gamma}}{\delta H^{a}(z) \delta c^{b}(x) \delta \bar{c}^{c}(y)}\right|_{H=\bar{c}=c=0}
\end{gathered}
$$

By replacing $\bar{\Gamma}$ with $W_{\text {local }}$ and taking into consideration the values of 
$\Pi_{\mu \nu}^{a b}(p), \Omega^{a b}(p)$ and $V^{a b c}\left(p_{1}, p_{2}\right)$ in eqs. (5.1), (6.1) and (6.3), we obtain:

$$
w_{1}=w_{1}^{(1)}=\frac{c_{V}}{k}, w_{2}=w_{2}^{(1)}=\frac{2}{3} \frac{c_{V}}{k}, \quad w_{3}=w_{3}^{(1)}=0 .
$$

Note that these values, together with $W_{\text {local }}$, fix completely the local contribution $\bar{\Gamma}_{\text {local }}^{(1)}$ to $\bar{\Gamma}^{(1)}$. The superscripts in $w_{1}^{(1)}, w_{2}^{(1)}$ and $w_{3}^{(1)}$ stand for "one-loop".

We next compute the local part $\bar{\Gamma}_{\text {local }}^{(2)}$ of the two-loop contribution $\bar{\Gamma}^{(2)}$ to the bare effective action. Again, terms with more than three fields are purely nonlocal, whereas those involving less than four fields are local. Thus, if $\bar{\Gamma}_{2}^{(2)}$ and $\bar{\Gamma}_{3}^{(2)}$ denote respectively the two- and three-field contributions to $\bar{\Gamma}^{(2)}$, we have

$$
\bar{\Gamma}_{\text {local }}^{(2)}=\bar{\Gamma}_{2}^{(2)}+\bar{\Gamma}_{3}^{(2)}
$$

In what follows we find $\bar{\Gamma}_{\text {local }}^{(2)}$ by solving eq. (8.9) up to order three in the number of fields. To do so we introduce the functional $Y_{3}$ :

$$
Y_{3}=\int d^{3} x\left[\frac{\delta \bar{\Gamma}_{\text {local }}^{(1)}}{\delta A^{\mu a}} \frac{\delta \bar{\Gamma}_{\text {local }}^{(1)}}{\delta G_{\mu}^{a}}+\frac{\delta \bar{\Gamma}_{\text {local }}^{(1)}}{\delta c^{a}} \frac{\delta \bar{\Gamma}_{\text {local }}^{(1)}}{\delta H^{a}}\right]
$$

with $\bar{\Gamma}_{\text {local }}^{(1)}$ given by eq. (8.17) with coefficients (8.21). It is a matter of some algebra to see that the functional $Y_{3}$ takes the form:

$$
Y_{3}=\frac{i k}{4 \pi}\left(\frac{c_{V}}{k}\right)^{2} \int d^{3} x \epsilon^{\mu \rho \nu} f^{a b c} A_{\mu}^{a} A_{\rho}^{b} \partial_{\nu} c^{c} .
$$

The key point now is to observe that $Y_{3}$ can be written as

$$
Y_{3}=\Delta_{1} \Upsilon_{2}
$$

where the functional $\Upsilon_{2}$ reads

$$
\Upsilon_{2}=-\frac{i k}{4 \pi}\left(\frac{c_{V}}{k}\right)^{2} \int d^{3} x \epsilon^{\mu \rho \nu} A_{\mu}^{a} \partial_{\rho} A_{\nu}^{a} .
$$

Eq. (8.9) then implies that $\bar{\Gamma}_{2}^{(2)}$ and $\bar{\Gamma}_{3}^{(2)}$ satisfy the following two equations:

$$
\Delta_{0} \bar{\Gamma}_{2}^{(2)}=0 \quad, \quad \Delta_{0} \bar{\Gamma}_{3}^{(2)}+\Delta_{1}\left[\Upsilon_{2}+\bar{\Gamma}_{2}^{(2)}\right]=0
$$

where we have used that $\Delta_{0} \Upsilon_{2}$ vanishes. Eqs. (8.24) are again of the type (8.15), 
with $W_{2}=\Upsilon_{2}+\bar{\Gamma}_{2}^{(2)}$ and $W_{3}=\bar{\Gamma}_{3}^{(2)}$, and are to be solved over the same space of functionals. We thus conclude that $\Upsilon_{2}+\bar{\Gamma}_{\text {local }}^{(2)}$ is of the form (8.17), or equivalently (8.18). The coefficients $w_{1}, w_{2}$ and $w_{3}$ can be calculated in the same way as before; for them we obtain:

$w_{1}=w_{1}^{(2)}=\frac{14}{3}\left(\frac{c_{V}}{k}\right)^{2} \quad, \quad w_{2}=w_{2}^{(2)}=\frac{169+L}{72}\left(\frac{c_{V}}{k}\right)^{2} \quad, \quad w_{3}=w_{3}^{(2)}=0$

with $L$ as in eq. (5.17). Putting everything together we have that the local part of the effective action $\Gamma_{\text {local }}$ up to second order in perturbation theory is:

$$
\begin{aligned}
\Gamma_{\text {local }}= & -\frac{i k}{4 \pi} \int d^{3} x \epsilon^{\mu \rho \nu}\left(\frac{1}{2} R_{1} A_{\mu}^{a} \partial_{\rho} A_{\nu}^{a}+\frac{1}{3 !} R_{2} f^{a b c} A_{\mu}^{a} A_{\rho}^{b} A_{\nu}^{c}\right) \\
& +\int d^{3} x\left[G_{\mu}^{a}\left(R_{3} \partial_{\mu} c^{a}-f^{a b c} c^{b} A^{\mu c}\right)-b^{a} \partial A^{a}-\frac{1}{2} f^{a b c} H^{a} c^{b} c^{c}\right] .
\end{aligned}
$$

where $R_{1}, R_{2}$ and $R_{3}$ are functions of $k^{-1}$ given by

$$
\begin{aligned}
& R_{1}=1+\frac{7}{3} \frac{c_{V}}{k}+\frac{265+L}{36}\left(\frac{c_{V}}{k}\right)^{2}, \\
& R_{2}=1+3 \frac{c_{V}}{k}+\frac{281+L}{24}\left(\frac{c_{V}}{k}\right)^{2}, \\
& R_{3}=1-\frac{2}{3} \frac{c_{V}}{k}-\frac{169+L}{72}\left(\frac{c_{V}}{k}\right)^{2} .
\end{aligned}
$$

To close this section we show that the double limit in eq. (8.1) exists up to two loops. We have seen in Sect. 7 that the limit $D \rightarrow 3$ of the dimensionally regularized TMYM effective action

$$
\Gamma(A, b, G, c, H ; k ; m, D)=\bar{\Gamma}(A, G, c, H ; k ; m, D)-\int d^{D} x b^{a} \partial A^{a}
$$

is finite at any order in perturbation theory. Hence, the limit $D \rightarrow 3$ in (8.1) exists. Moreover, we know that both dimensional regularization methods in Sect. 2 yield the same result as $D$ goes to 3 and that this limit is BRS-invariant. This 
means that the bare TMYM action $\bar{\Gamma}(m)$ in (8.2) satisfies the BRS equation (8.4). In particular, its one-loop contribution must satisfy eq. (8.11), and so must the corresponding one-loop divergent part as $m$ goes to infinty (if any). Thus, if there were a one-loop large $m$ divergent contribution, it would be of the form (8.17), with $w_{1}, w_{2}$ and $w_{3}$ carrying large $m$ singularities. However, these coefficients can not be but zero, for the vacuum polarization tensor, the ghost self-energy and the Hcc vertex are finite as $m$ goes to infinity. We then infer that the double limit in eq. (8.1) exists at one loop. This, together with eq. (8.9), implies that the would-be two-loop large $m$ divergent contribution to $\bar{\Gamma}(m)$ would satisfy eq. (8.11) again. The same argument as for the one-loop order leads to large $m$ finiteness at two loops. Thus, our regularization method provides an UV finite CS effective action up to second order in perturbation theory in the least.

\section{Absence of two-loop corrections to Witten's shift.}

The purpose of this section is to show that two-loop contributions to the bare effective action in eq. (8.25) do not modify Witten's shift $k \rightarrow k+c_{V}$, where $k>0$ is the classical CS parameter [6]. We will see in particular that twoloop contributions correspond to a cohomologically trivial term in the sense of the cohomology of the Slavnov-Taylor operator. Hence, they are equivalent to a wave function renormalization of the fields and can be set to zero by an appropriate rescaling of the latter. Let us recall that in quantum field theory [43] the values of the observables are not modified by finite rescalings of the fields. Furthermore, the need of introducing wave function renormalizations to bring out the physical effects of radiative corrections to $1 \mathrm{PI}$ functions is a common feature in quantum field theory. Think, for instance, of the computation of the beta function from vertex radiative corrections.

We begin by showing how our formalism reproduces the one-loop shift. As already mentioned, the effective action (8.25) can be recast, up to first order in 
perturbation theory, into the form:

$$
\begin{aligned}
\Gamma_{\text {local }}(A, b, c, G, H ; k)= & -\frac{i\left(k+c_{V}\right)}{4 \pi} S_{C S}(A)-\int d^{3} x b^{a} \partial A^{a}+\Delta X_{\mathrm{gf}}+\Delta X \\
& + \text { two-loop contributions },
\end{aligned}
$$

with

$$
X_{\mathrm{gf}}=-\int d^{3} x H^{a} c^{a}, \quad X=\frac{2}{3} \frac{c_{V}}{k} \int d^{3} x G_{\mu}^{a} A^{a \mu},
$$

$\Delta$ the Slavnov-Taylor operator (8.10) and $G_{\mu}^{a}$ as in (8.5).

We already see from eq. (9.1) that the effect of first order radiative corrections is twofold. First, there is the shift of the classical parameter $k$ in front of the classical CS action $S_{C S}$. This is the one-loop shift first computed by Witten [6] and by several authors afterwards [19-22]. Notice that $S_{C S}$ is the only gaugeinvariant part of the functional (9.1), which in turn generates the local cohomology of the opertor $\Delta$ upon multiplication by $c$-numbers. Secondly, there is the term $\Delta X$. This term is gauge-dependent, as illustrates the fact that it vanishes in the background field gauge [21] and depends on the light-cone vectors in the lightcone gauge[32]. The contribution $\Delta X$ is both BRS-invariant, for the operator $\Delta$ annihilates it, and cohomologically trivial with respect to $\Delta$. Since the SlavnovTaylor operator $\Delta$ can be considered the quantum generalization of the classical BRS operator $s$ in (2.5), one would then not marvel if $\Delta X$ did not contribute to gauge-invariant quantities. The cohomological triviality of $\Delta X$ implies that there exists a wave function renormalization of the fields

$A_{\mu}^{a}=Z_{A} A_{\mu}^{\prime a}, b^{a}=Z_{b} b^{a}, G_{\mu}^{a}=Z_{G} G_{\mu}^{a}, c^{a}=Z_{c} c^{a}, \quad H^{a}=Z_{H} H^{\prime a}$,

with

$$
Z_{A}=Z_{G}^{-1}=Z_{b}^{-1} \quad, \quad Z_{c}=Z_{H}^{-1}
$$

such that if $\Gamma^{(0)}(\Phi, k)$ denotes the tree-level action, the following equation holds 
at one loop:

$$
\Delta X=\Gamma^{(0)}\left(Z_{\Phi}^{-1} \Phi, k\right)-\Gamma^{(0)}(\Phi, k)
$$

This equation holds for the more general $X$ and $W_{\text {local }}$ of eqs. (8.19) and (8.18), and for the values in (8.21) it yields:

$$
Z_{A}=Z_{G}^{-1}=Z_{b}^{-1}=1-\frac{2}{3} \frac{c_{V}}{k}, Z_{c}=Z_{H}^{-1}=1
$$

Indeed, this wave function renormalization transforms the action (9.1) into

$$
\begin{aligned}
\Gamma_{\text {local }}\left(A^{\prime}, b^{\prime}, c^{\prime}, G^{\prime}, H^{\prime} ; k\right)= & -\frac{i\left(k+c_{V}\right)}{4 \pi} S_{C S}\left(A^{\prime}\right) \\
& +\int d^{3} x\left[-b^{\prime a} \partial A^{\prime a}+G^{\prime a \mu} D_{\mu}^{a b}\left(A^{\prime \prime}\right) c^{\prime b}-\frac{1}{2} f^{a b c} H^{\prime a} c^{\prime b} c^{\prime c}\right] \\
& + \text { two-loop contributions . }
\end{aligned}
$$

We thus see that the shift in $k$ is all that remains after the rescaling of the fields (9.2). The fact that $Z_{c}$ does not receive radiative contributions is a special feature of Landau gauges[53].

The properties of $\Delta X$ just described imply that it does not contribute to the vacuum expectation values of Wilson loops. To prove that this is the case lies outside the scope of this paper; it is a technical problem that will be reported elsewhere [54]. Suffice it to say that the gauge field contributions in $\Delta X$ cancel against the large $m$ contributions coming from the integrals

$$
\begin{gathered}
\int_{0}^{1} d t_{1} \int_{0}^{t_{1}} d t_{2} \int_{0}^{t_{2}} d t_{3} \dot{x}^{\mu}\left(t_{1}\right) \dot{x}^{\rho}\left(t_{2}\right) \dot{x}^{\nu}\left(t_{3}\right)<A_{\mu}\left(t_{1}\right) A_{\rho}\left(t_{2}\right) A_{\nu}\left(t_{3}\right)> \\
\int_{0}^{1} d t_{1} \int_{0}^{t_{1}} d t_{2} \int_{0}^{t_{2}} d t_{3} \int_{0}^{t_{3}} d t_{4} \dot{x}^{\mu}\left(t_{1}\right) \dot{x}^{\rho}\left(t_{2}\right) \dot{x}^{\nu}\left(t_{3}\right) \dot{x}^{\sigma}\left(t_{4}\right)<A_{\mu}\left(t_{1}\right) A_{\nu}\left(t_{3}\right)><A_{\rho}\left(t_{2}\right) A_{\sigma}\left(t_{4}\right)>
\end{gathered}
$$

wherever two gauge fields and three gauge fields, respectively, lie arbitrarily close to each other on the Wilson line. The Green functions inside these line integrals 
are computed for finite $m$. We would like to stress that this cancelation is not accidental but a consequence of BRS invariance, namely, that the one-loop gaugedependent contributions to $\Gamma_{\text {local }}$ are of the cohomologically trivial form $\Delta X$. The cancelation mechanism goes beyond CS theory and is implied by BRS invariance [55], this symmetry being explicitly preserved in our formalism.

Let us now consider second order radiative corrections. First, we substract from eq. (8.25) two-loop corrections arising from the one-loop contribution in $\Delta X$ that enters the two-loop diagrams through one-loop subdiagrams. This is achieved by means of the wave function renormalization (9.2). In terms of the renormalized fields, the local part of the effective action reads:

$$
\begin{aligned}
\Gamma_{\text {local }}\left(A^{\prime}, b^{\prime}, c^{\prime}, G^{\prime}, H^{\prime} ; k\right) & =-\frac{i\left(k+c_{V}\right)}{4 \pi} S_{C S}\left(A^{\prime}\right) \\
& +\int d^{3} x\left[-b^{\prime a} \partial A^{\prime a}+G^{\prime a \mu} D_{\mu}^{a b}\left(A^{\prime}\right) c^{\prime b}-\frac{1}{2} f^{a b c} H^{\prime a} c^{\prime b} c^{\prime c}\right] \\
& -c_{2} \int d^{3} x\left[\frac{i k}{4 \pi} \epsilon^{\mu \rho \nu}\left(A_{\mu}^{\prime a} \partial_{\rho} A_{\nu}^{\prime a}+\frac{1}{2} f^{a b c} A_{\mu}^{\prime a} A_{\rho}^{\prime b} A_{\nu}^{\prime c}\right)+G^{\prime a \mu} \partial_{\mu} c^{\prime a}\right] \\
& + \text { three-loop contributions },
\end{aligned}
$$

with

$$
c_{2}=\frac{169+L}{72}\left(\frac{c_{V}}{k}\right)^{2}
$$

and $L$ as in (5.17). The functional (9.3) can be recast as

$$
\begin{aligned}
\Gamma_{\text {local }}\left(A^{\prime}, b^{\prime}, c^{\prime}, G^{\prime}, H^{\prime} ; k\right)= & -\frac{i\left(k+c_{V}\right)}{4 \pi} S_{C S}\left(A^{\prime}\right)-\int d^{3} x b^{\prime a} \partial A^{\prime a}+\Delta^{\prime} X_{\mathrm{gf}}^{\prime}+\Delta^{\prime} X^{\prime} \\
& + \text { three-loop contributions }
\end{aligned}
$$

where

$$
X_{\mathrm{gf}}^{\prime}=-\int d^{3} x H^{\prime a} c^{\prime a}, \quad X^{\prime}=c_{2} \frac{c_{V}}{k} \int d^{3} x G_{\mu}^{\prime a} A^{\prime a \mu}
$$

and $\Delta^{\prime}$ is the Slavnov-Taylor operator in terms of $A_{\mu}^{\prime a}, c^{\prime a}, G_{\mu}^{\prime a}$ and $H^{\prime a}$, obtained 
from $\Delta$ in eq. (8.10) by replacing every field with its primed counterpart. We see in eq. (9.4) that, once the one-loop wave function renormalization (9.2) has been performed, all second order radiative corrections fit into the cohomologically trivial functional $\Delta^{\prime} X^{\prime}$ in the sense of the cohomology of $\Delta^{\prime}$. Notice that the coefficient in front of $S_{C S}\left(A^{\prime}\right)$ does not receive any two-loop contribution. Upon multiplication by $c$-numbers, the functional $S_{C S}\left(A^{\prime}\right)$ generates the local cohomology of $\Delta^{\prime}$ over the space of integrated local functionals of mass dimension three and ghost number zero.

The fact that the wave function renormalization (9.2) leads to the effective action (9.4) can be partially understood by using BRS invariance. To see this we first notice that the effect of the renormalization (9.2) on the two-loop BRS equation (8.9) is to cancel the contribution $Y_{3}$ of eq. (8.23) so that eq. (8.9) leads to the following equation for $\bar{\Gamma}_{\text {local }}^{(2)}\left(A^{\prime}, c^{\prime}, G^{\prime}, H^{\prime} ; k\right)$ :

$$
\Delta^{\prime} \bar{\Gamma}_{\text {local }}^{(2)}\left(A^{\prime}, c^{\prime}, G^{\prime}, H^{\prime} ; k\right)=0
$$

The most general solution of this equation is

$$
-\frac{i \gamma}{4 \pi} S_{C S}\left(A^{\prime}\right)+\Delta^{\prime} Y^{\prime}
$$

where $Y^{\prime}$ is given by eq. (8.19) with every field replaced with its primed counterpart. Hence, if $\gamma$ is equal to zero, the functional $\bar{\Gamma}_{\text {local }}^{(2)}\left(A^{\prime}, c^{\prime}, G^{\prime}, H^{\prime} ; k\right)$ can not have but the form $\Delta^{\prime} Y^{\prime}$. This is precisely what happens in our case, and thus, eq. (9.4) is in part a consequence of the BRS invariance of the renormalized action. We say in part because the piece of information $\gamma=0$ is not provided by BRS invariance. On the contrary, it results from our computations in previous sections. Did $\gamma$ not vanish, there would be a two-loop correction $\gamma$ to Witten's one-loop shift $k \rightarrow k+c_{V}$.

Again, the cohomological triviality of $\Delta^{\prime} X^{\prime}$ implies that the latter can be 
absorbed by the following two-loop renormalization of the fields:

$$
A^{\prime a \mu}=Z_{A^{\prime}} A_{R}^{a \mu}, b^{\prime a}=Z_{b^{\prime}} b_{R}^{a}, G^{\prime a \mu}=Z_{G^{\prime}} G_{R}^{a \mu}, c^{\prime a}=Z_{c^{\prime}} c_{R}^{a}, H^{\prime a}=Z_{H^{\prime}} H_{R}^{a},
$$

with

$$
Z_{A^{\prime}}=Z_{G^{\prime}}^{-1}=Z_{b^{\prime}}^{-1}=1-\frac{169+L}{72}\left(\frac{c_{V}}{k}\right)^{2}, \quad Z_{c^{\prime}}=Z_{H^{\prime}}^{-1}=1
$$

We also have that if $\Gamma^{(0)}\left(\Phi^{\prime}, k\right)$ is the tree-level action in terms of the fields $A_{\mu}^{\prime a}, b^{\prime a}, c^{\prime a}, G_{\mu}^{\prime a}$ and $H^{\prime a}$, the identity

$$
\Delta^{\prime} X^{\prime}=\Gamma^{(0)}\left(Z_{\Phi^{\prime}}^{-1} \Phi^{\prime}, k\right)-\Gamma^{(0)}\left(\Phi^{\prime}, k\right)
$$

holds at the order we are working. The local part of the effective action in terms of the fields $A_{R}^{a \mu}, b_{R}^{a}, c_{R}^{a}, G_{R}^{a \mu}$ and $H_{R}^{a}$ takes the form:

$$
\begin{aligned}
\Gamma_{\text {local }}\left(A_{R}, b_{R}, c_{R}, G_{R}, H_{R} ; k\right)= & -\frac{i\left(k+c_{V}\right)}{4 \pi} S_{C S}\left(A_{R}\right) \\
& +\int d^{3} x\left[-b_{R}^{a} \partial A_{R}^{a}+G_{R}^{a \mu} D_{\mu}^{a b}\left(A_{R}\right) c_{R}^{b}-\frac{1}{2} f^{a b c} H_{R}^{a} c_{R}^{b} c_{R}^{c}\right] \\
& + \text { three-loop contributions . }
\end{aligned}
$$

The parameter in front of the gauge-invariant part $S_{C S}$ is still the one-loop parameter $k+c_{V}$. This shows that there are no second order radiative corrections to the one-loop shift of the classical parameter $k$. In other words, local two-loop corrections in the bare effective action (8.25) correspond to a wave function renormalization and are, therefore, unobservable [43]. The existence of such renormalization follows from the cohomologically trivial form $\Delta^{\prime} X^{\prime}$ in which the two-loop contributions can be written and can be seen as a consequence of BRS invariance. The rationale why $\Delta^{\prime} X^{\prime}$ should not contribute to the vacuum expectation values of Wilson loops is very much the same as for $\Delta X$, roughly scketched above. The 
outcome of this discussion is that still after taking into account second order radiative corrections, the monodromy parameter is $k+c_{V}$. This result is in agreement with Wilson loop computations for the unknot [34], which demand that two-loop corrections to the vacuum polarization tensor do not contribute to the Wilson loop, if Witten's exact result is to be recovered in perturbation theory.

We finish this section by collecting the wave function renormalization that transforms the local effective action (8.25) into the form (9.5):

$$
\begin{aligned}
& Z_{b}^{-1}=Z_{G}^{-1}=Z_{A}=1-\frac{2}{3} \frac{c_{V}}{k}-\left(\frac{169}{72}+\frac{22}{3} \ln 2-\frac{63}{8} \ln 3\right)\left(\frac{c_{V}}{k}\right)^{2} . \\
& Z_{c}=Z_{H}^{-1}=1 .
\end{aligned}
$$

Both actions (8.25) and (9.5) yield the same values for the observables of the theory. However, the effective action (9.5) clearly displays what the radiative corrections contributing to the observables are.

\section{Conclusions}

Working in the covariant Landau gauge, we have studied CS theory as the large mass limit of TMYM theory, the latter being defined as the standard Yang-Mills theory in three dimensions with a CS term responsible for a topological mass $m$. The idea behind this approach is to use the Yang-Mills term as a higher-covariant derivative regularization so that CS quantities are computed by sending $m$ to infinity in their TMYM counterparts. However, this is not good enough, since TMYM theory is not finite by power counting and, hence, the large $m$ limit can not be taken directly. One first has to introduce an additional regulator to cure TMYM divergences. Here we have used dimensional regularization and have shown that it is BRS-invariant. Our approach to CS theory can then be viewed as a hybrid BRS-invariant regularization consisting of a higher covaraint derivative Yang-Mills term plus dimensional regularization. In particular, bare CS Green functions are defined as the limit $m \rightarrow \infty$ of the limit $D \rightarrow 3$ of the corresponding dimensionally regularized TMYM Green functions. 
We have seen that the limit $D \rightarrow 3$ of any dimensionally regularized TMYM Green function is well-defined at any perturbative order. This shows that TMYM theory is perturbatively finite and guarantees that taking the limit $m \rightarrow \infty$ is legitimate. To compute the latter limit we have given two large-mass vanishing theorems. One is thus left with all necessary tools to calculate. In this paper we have computed the local part of the CS effective action up to second order in perturbation theory. We have done this by solving, up to two loops, the BRS identities for the effective action over the space of local functionals. The coefficients entering in the solution can be determined by exploiting that the effective action generates 1PI Green functions. This demands computing three 1PI Green functions. Here we have chosen the vacuum polarization tensor, the ghost self-energy and the ghost-ghost-external field vertex. Their one- and two-loop expressions are given in (5.1), (5.16), (6.1) -(6.3) and (6.5), which in turn yield for the bare effective action eq. (8.25).

To construct the quantum theory, regularization is not enough but has to be supplemented with renormalization conditions. Notice that the theory being finite does not necessarily imply that an UV finite renormalization is not needed. As a matter of fact, there are many finite renormalizations leading to different expressions for the renormalized effective action and the observables (see [35] for examples). Of course, for a finite theory like ours, one can always choose a renormalization scheme in which the renormalized coupling constant and fields are equal to the bare ones, but this is already a choice. The problem is that there are no arguments within the framework of local and renormalized perturbative quantum field theory that lead naturally to a renormalization scheme. The reasons for a given parametrization have to be found outside this framework. For finite theories one may assume that the classical parameters constitute the right parametrization, provided a regularization preserving the symmetries of the observables or fundamental symmetries is used[56]. The idea is that the fundamental symmetries, when respected at the regularized level, are strong enough as to fix all ambiguities with observables effects introduced by regularization. For this parametrization to make 
sense, the emerging quantum theory has to be independent of the particular invariant regularization one uses; or in other words, all regularizations preserving the fundamental symmetries have to give the same values for the observables as functions of the bare parameters. In the case at hand, the only symmetry of the observables is gauge invariance, which in the gauge-fixed theory becomes BRS invariance. Furthermore, it is known that all BRS-invariant regularizations of CS theory give the same result for the expectation values of Wilson loops in terms of the bare or classical $k$, at least at first order in perturbation theory [19-22]. In addition, also at this order, the values in terms of the bare $k$ for the monodromy parameter of the observables and for the central extension of the 2-dimensional current algebra are the same as for the hamiltonian formalism [15]. Based upon these two facts we have parametrized the theory in terms of the bare or classical $k$.

With this choice of parametrization we have finally analyzed the relevance of the radiative corrections we have computed. In gauge theories, only gauge-invariant (rather than merely BRS-invariant) corrections contribute to the observables. To extract gauge-invariant radiative corrections from the local effective action, we have studied the cohomology of the Slavnov-Taylor operator, since contributions cohomologically trivial do not contribute to the observables. As a result, we have obtained for the effective action that:

i) Part of one-loop radiative corrections are gauge-invariant and produce the shift $k \rightarrow k+c_{V}$ of the bare CS parameter $k$, in agreement with previous results in the literature [6,19-22]. The remaining oneloop contributions are gauge-dependent. Indeed, they are cohomologically trivial with respect to the Slavnov-Taylor operator so that they can be set to zero by means of a finite multiplicative wave function renormalization of only the fields. Hence, they do not contribute to the observables of the theory [54], in agreement with the general statement that UV finite renormalizations of the fields are unobservable [43]. 
ii) All two-loop radiative corrections are cohomologically trivial in the sense of the Slavnov-Taylor operator, hence gauge-dependent. They can thus be set to zero by renormalizing the fields. Two-loop corrections to the effective action do not contribute therefore to the observables of the theory. This means that the shift $k \rightarrow k+c_{V}$ does not receive second order contributions.

We would like to mention that the analysis presented here clearly displays what the options are. If one parametrizes the quantum theory in terms of the classical $k$, the one-loop shift $k \rightarrow k+c_{V}$ does not receive two-loop contributions. If, on the other hand, one insists in a renormalized CS parameter $k_{R}$, it does not make any sense to speak of a shift. Obviously, one can tunnel between these two situations by a finite renormalization $k_{R}=k+c_{V}$. We think, however, that the fact that all BRS-invariant regularizations tried in the literature give the same shift in $k$ when the quantum theory is parametrized in terms of the classical CS parameter is no accident, and that there must be a fundamental reason for that. Let us stress that the latter does not mean that the one-loop Landau-gauge bare effective action should be the same for all BRS-invariant regularizations, since the effective action is not an observable itself. It rather means that the corrections in the effective action that contribute to the observables of the theory should be the same for all BRS-invariant regularizations. This is what one actually observes in the existing literature: gauge-invariant, or equivalently cohomologically non-trivial one-loop corrections to the effective action are the same for any BRS-preserving regularization and provide the famous shift. The remaining one-loop corrections to the bare effective action depend on the BRS-invariant regularization one uses. However, they fit into a cohomologically trivial functional, i.e. they correspond to wave function renormalizations of only the fields and are therefore unobservable. To summarize, bare Green functions provided by different BRS-invariant regulators differ as much as possible in so far as the shift of the classical parameter remains unchanged. According to the general principles of local and renormalized perturbation theory [52], these differences should even reach the shift, unless there 
is some reason why they should not.

Let us close by anticipating what three-loop radiative corrections should be, if the pattern we have unveiled occurs beyond two loops. Once one- and twoloop cohomologically trivial contributions have been absorbed by appropriate wave function renormalizations, all three-loop corrections should thoroughly fit into a cohomologically trivial functional with respect to the renormalized Slavnov-Taylor operator. The value of these corrections will in general depend on the particular BRS-invariant regulator used. There should be however no contributions to the one-loop shift.

$\underline{\text { Acknowledgements: }}$

The authors are grateful to Professors G. Leibbrandt and J.C. Taylor for valuable conversations and encouragement. GG was supported by the Italian Consiglio Nazionale delle Ricerche, CPM by the National Science and Engineering Research Council of Canada under grant No. A-8063, and FRR by The Commission of the European Communities through contract No. SC1000488 with The Niels Bohr Institute. Their work also benefited from partial support from CICYT, Spain. 


\section{APPENDIX}

We give here a list of integrals that have to be computed to obtain the explicit expressions for the gauge field polarization tensor and the ghost self-energy of eqs. (5.16) and (6.2). As explained in Sect. 5, the use of some algebraic identities and of the $m$ - and $o$-theorems reduces the problem to that of calculating a finite number of one- and two-loops integrals. One-loop integrals are of the form

$$
\int \frac{d^{D} q}{(2 \pi)^{D}} \frac{1}{\left(q^{2}\right)^{\alpha}\left(q^{2}+1\right)^{\beta}}=\frac{\Gamma\left(\frac{D}{2}-\alpha\right) \Gamma\left(\alpha+\beta-\frac{D}{2}\right)}{(4 \pi)^{D / 2} \Gamma(\beta) \Gamma\left(\frac{D}{2}\right)} .
$$

As for the two-loop integrals they can be classified into four types, namely:

$$
\begin{aligned}
I_{\alpha \beta \gamma}^{(0)} & =\int \frac{d^{D} k}{(2 \pi)^{D}} \frac{d^{D} q}{(2 \pi)^{D}} \frac{1}{\left(k^{2}\right)^{\alpha}\left(q^{2}\right)^{\beta}\left[(k+q)^{2}\right]^{\gamma}}, \\
I_{\alpha \beta \gamma}^{(1)} & =\int \frac{d^{D} k}{(2 \pi)^{D}} \frac{d^{D} q}{(2 \pi)^{D}} \frac{1}{\left(k^{2}+1\right)^{\alpha}\left(q^{2}\right)^{\beta}\left[(k+q)^{2}\right]^{\gamma}}, \\
I_{\alpha \beta \gamma}^{(2)} & =\int \frac{d^{D} k}{(2 \pi)^{D}} \frac{d^{D} q}{(2 \pi)^{D}} \frac{1}{\left(k^{2}+1\right)^{\alpha}\left(q^{2}+1\right)^{\beta}\left[(k+q)^{2}\right]^{\gamma}}, \\
I_{\alpha \beta \gamma}^{(3)} & =\int \frac{d^{D} k}{(2 \pi)^{D}} \frac{d^{D} q}{(2 \pi)^{D}} \frac{1}{\left(k^{2}+1\right)^{\alpha}\left(q^{2}+1\right)^{\beta}\left[(k+q)^{2}+1\right]^{\gamma}} .
\end{aligned}
$$

In dimensional regularization integrals of the first type vanish

$$
I_{\alpha \beta \gamma}^{(0)}=0
$$

To evaluate $I_{\alpha \beta \gamma}^{(1)}$ and $I_{\alpha \beta \gamma}^{(2)}$ we use the method of Feynman parameters. It is straightforward to show that

$$
I_{\alpha \beta \gamma}^{(1)}=\frac{\Gamma\left(\frac{D}{2}-\beta\right) \Gamma\left(\frac{D}{2}-\gamma\right) \Gamma\left(\beta+\gamma-\frac{D}{2}\right) \Gamma(\alpha+\beta+\gamma-D)}{(4 \pi)^{D} \Gamma(\alpha) \Gamma(\beta) \Gamma(\gamma) \Gamma\left(\frac{D}{2}\right)}
$$

The computation of $I_{\alpha \beta \gamma}^{(2)}$ is however more involved. We next present some of its 
details. Integrating over $q$ we obtain:

$$
\begin{aligned}
I_{\alpha \beta \gamma}^{(2)}=\frac{\Gamma\left(\gamma+\beta-\frac{D}{2}\right)}{(4 \pi)^{D / 2} \Gamma(\gamma) \Gamma(\beta)} & \int_{0} \frac{d^{D} k}{(2 \pi)^{D}}\left(k^{2}+1\right)^{\frac{D}{2}-\alpha-\beta-\gamma} \\
& \times \int_{0}^{1} d x x^{\frac{D}{2}-\gamma-1}(1-x)^{\gamma-1}\left(1-\frac{k^{2}}{k^{2}+1} x\right)^{\frac{D}{2}-\gamma-\beta} .
\end{aligned}
$$

Integration over $x$ gives [57]:

$$
B\left(\frac{D}{2}-\gamma, \gamma\right) F\left(\gamma+\beta-\frac{D}{2}, \frac{D}{2}-\gamma ; \frac{D}{2}, \frac{k^{2}}{k^{2}+1}\right)
$$

where $B$ is Euler's beta function and $F$ is Gauss' hypergeometric function. To integrate over $k$ we use polar coordinates and perform the change of variables $t=\frac{k^{2}}{k^{2}+1}$. In this way we get

$$
\begin{aligned}
I_{\alpha \beta \gamma}^{(2)}= & \frac{\Gamma\left(\gamma+\beta-\frac{D}{2}\right) \Gamma\left(\frac{D}{2}-\gamma\right)}{(4 \pi)^{D} \Gamma(\beta)\left[\Gamma\left(\frac{D}{2}\right)\right]^{2}} \\
& \quad \times \int_{0}^{1} d t t^{\frac{D}{2}-1}(1-t)^{\alpha+\beta+\gamma-D-1} F\left(\gamma+\beta-\frac{D}{2}, \frac{D}{2}-\gamma ; \frac{D}{2}, t\right) .
\end{aligned}
$$

The integral on the RHS can be performed [57] and gives a certain product of Euler's gamma functions so that

$$
I_{\alpha \beta \gamma}^{(2)}=\frac{\Gamma\left(\gamma+\beta-\frac{D}{2}\right) \Gamma\left(\frac{D}{2}-\gamma\right) \Gamma(\alpha+\beta+\gamma-D) \Gamma\left(\alpha+\gamma-\frac{D}{2}\right)}{(4 \pi)^{D} \Gamma(\alpha) \Gamma(\beta) \Gamma(\alpha+\beta+2 \gamma-D) \Gamma\left(\frac{D}{2}\right)} .
$$

It should be stressed that all these manipulations are in the sense of dimensional regularization. More precisely, they hold for $D, \alpha, \beta$ and $\gamma$ in suitable domains. To obtain the value of $I_{\alpha \beta \gamma}^{(2)}$ outside them one uses analytic continuation. This same observation applies to all the integrals in this appendix. 
For $I_{\alpha \beta \gamma}^{(3)}$ we have not found a compact expression in terms of the parameters $D, \alpha, \beta$ and $\gamma$. However, the result does look very simple for specific values of them. Here we list all integrals of this type at $D=3$ encountered when performing the calculations of Sects. 5 and 6:

$$
\begin{gathered}
I_{111}^{(3)}=\frac{1}{32 \pi^{2}}\left\{-\frac{1}{\varepsilon}+1-\gamma+\ln \left(\frac{4 \pi}{9}\right)+O(\varepsilon)\right\} \\
I_{121}^{(3)}=\frac{1}{96 \pi^{2}} \quad, \quad I_{131}^{(3)}=\frac{1}{288 \pi^{2}} \quad, \quad I_{221}^{(3)}=\frac{1}{576 \pi^{2}} \quad, \quad I_{231}^{(3)}=\frac{5}{6912 \pi^{2}},
\end{gathered}
$$

where $\varepsilon=D-3$ is the regulator.

Note that $I_{111}^{(1)}, I_{111}^{(2)}$ and $I_{111}^{(3)}$ are overall UV divergent at $D=3$, exhibiting a pole at $\varepsilon=0$. 


\section{REFERENCES}

1. R. Jackiw and S. Templeton, Phys. Rev. D23 (1981) 2291.

2. J. Schonfeld Nucl. Phys. B185 (1981) 157.

3. S. Deser, R. Jackiw and S. Templeton, Ann. Phys. 140 (1982) 372.

4. S. Deser, R. Jackiw and S. Templeton, Phys. Rev. Lett. 48 (1982) 975.

5. R. Pisarski and S. Rao, Phys. Rev. D32 (1985) 2081.

6. E. Witten, Commun. Math. Phys. 121 (1989) 351.

7. E. Witten, Commun. Math. Phys. 17 (1988) 353.

8. D. Birmingham, M. Blau, M. Rakowski and G. Thompson, Topological field theory, Phys. Rep., to appear.

9. G. Moore and N. Seiberg, Phys. Lett. B220 (1989) 422.

10. J. Frölich and U. Studer, $U(1) \times S U(2)$-gauge invariance of non-relativistic quantum mechanics and generalized Hall effects, ETH-TH/91-13 preprint.

11. V.F.R Jones, Ann. Math. 126 (1987) 335.

12. P. Freyd, D. Yetter, J. Hoste, W.B.R. Lickorish, K. Millet and O. Ocneanu, Bull. Am. Math. Soc. 12 (1985) 239.

13. S. Elitzur, G. Moore, A. Schwimmer and N. Seiberg, Nucl. Phys. B326 (1989) 108.

T. Killingback, Phys. Lett. B219 (1989) 448.

G.V. Dunne, R. Jackiw and C.A. Trugenberger, Ann. Phys. 194 (1989) 197.

M. Bos and V.P. Nair, Int. J. Mod. Phys. A5 (1990)959.

J.M.F. Labastida, P.M. Llatas and A.V. Ramallo, Nucl. Phys. B348 (1991) 651.

14. J. Fröhlich and C. King, Commun. Math. Phys. 126 (1989) 167. 
15. L. Alvarez-Gaumé, J.M.F. Labastida and A.V. Ramallo, Nucl. Phys. B, Proc. Suppl. 18B (1990) 1.

16. E. Guadagnini, M. Martellini and M. Mintchev, Phys. Lett. 224 (1989) 489; Nucl. Phys. B330 (1990) 575.

P. Cotta-Ramusino, E. Guadagnini and M. Mintchev, Nucl. Phys. B330 (1990) 577.

17. L. Kauffman, On Knots, Princeton University Press (1987).

18. E. Guadagnini, M. Martellini and M. Mintchev, Phys. Lett. B227 (1989) 111.

19. L. Alvarez-Gaumé, J.M.F. Labastida and A.V. Ramallo, Nucl. Phys. B334 (1990) 103.

20. M. Asorey and F. Falceto, Phys. Lett. 241 (1990) 31.

21. C.P. Martin, Phys. Lett. B241 (1990) 513.

22. W. Chen, G.W. Semenoff and Yong-Shi Wu, Mod. Phys. Lett. A5 (1990) 1833.

23. D. Birmingham, R. Kantwoski and M. Rakowski, Phys. Lett. B251 (1990) 121.

24. C.P. Martin, Phys. Lett. B246 (1991) 87.

25. G.P. Korchemsky, Mod. Phys. Lett. A6 (1990) 727.

26. D. Birmingham, M. Rakowski and G. Thompson, Nucl. Phys. B234 (1990) 367.

F. Delduc, F. Gieres and S.P. Sorella, Phys. Lett. B225 (1989) 367.

P.H. Damgaard and V.O. Rivelles, Phys. Lett. B245 (1989) 48.

C. Lucchesi and O. Piguet, Supersymmetry of the Chern-Simons theory on a general three-dimensional manifold, MPI-PAE/Pth 20/91 and UGVA-DPT 1991/04-713 preprints. 
27. A. Blasi and R. Collina, Nucl. Phys. B345 (1990) 472.

28. F. Delduc, C, Lucchesi, O. Piguet and S.P. Sorella, Nucl. Phys. B346 (1990) 513.

29. D. Daniel and N. Dorey, Phys. Lett B246 (1990) 82.

N. Dorey, Phys. Lett. B246 (1990) 87.

30. M.A. Shifman, Nucl. Phys. B352 (1991) 87.

31. D. Bar-Natan, Perturbative Chern-Simons theory, Princeton University preprint 1990.

32. G. Leibbrandt and C.P. Martin, Perturbative Chern-Simons theory in the light-cone gauge, CERN-TH.6180/91 preprint.

33. S. Axelrod and I.M. Singer, Chern-Simons perturbation theory, MIT preprint 1991.

34. M. Alvarez and J.M.F. Labastida, Analysis of observables in Chern-Simons perturbation theory, US-FT-10/91 preprint.

35. G. Giavarini, C.P. Martin and F. Ruiz Ruiz, Supersymmetry breaking in a BRS-invariant regularization of Chern-Simons theory, LPTHE 91/49, NBI-HE 91-48 and UGMS 91/24 preprints.

36. R. Jackiw, Topics in Planar Physics, in Integrability and quantization, M. Asorey, J. F. Cariñena and L. A. Ibort eds., Nucl. Phys. B, Proc. Suppl. 18A (1990) 107.

37. G. Dunne, R. Jackiw and C.A. Trugenberger, Phys. Rev D 41 (1990) 661.

38. L.D. Faddeev and A.A. Slavnov, Gauge fields: introduction to quantum theory. The Benjamin Cummings Publishing Company (1980).

39. G. 't Hooft and M. Veltman, Nucl. Phys. B44 (1972) 189.

40. P. Breitenlohner and D. Maison, Commun. Math. Phys. 52 (1977) 11.

41. M. Bos, Ann. Phys. 181 (1988)197. 
42. H. Osborn, Ann. Phys. 200 (1990) 1.

43. J. C. Collins, Renormalization, Cambridge University Press (1987).

44. J. H. Lowenstein and W. Zimmerman, Comm. Math. Phys. 44 (1975) 73.

45. E. R. Speer, Ann. Inst. Henri Poincaré XXII (1975) 1.

E. R. Speer J. Math. Phys. 15 (1974) 1.

46. G. 't Hooft and M. Veltman, Nucl. Phys. B44(1972) 11.

47. E. R. Speer, Dimensional and analytic renormalization, in Renormalization theory, G. Velo and A.S. Wightman eds., Reidel Dordrecht (1976).

48. K. Hepp, Renormalization theory in Statistical mechanics and quantum field theory, Les Houches 1970, C. DeWitt and R. Stora eds., Gordon and Breach (1971).

H. Epstein and V. Glaser, Ann. Inst. Henri Poincaré XIX 3 (1973) 211.

D. Maison, Renormalization theory, a short account of results and problems in Renormalization of quantum field theories with non-linear field transformations, P. Breitenlohner, D. Maison and K. Sibold eds., Springer Verlag (1987).

49. S. Weinberg, Phys. Rev. 188 (1960) 838.

50. J.H. Lowenstein, BPHZ renormalization in Renormalization theory, G. Velo and A. S. Wightman eds., D. Reidel Publishing Company (1976).

51. M. Bos, private communication.

52. K. Hepp, Théorie de la renormalisation, Lectures Notes in Physics vol. 2, Springer-Verlag, Berlin (1969).

53. O. Blasi, O. Piguet and S.P. Sorella, Nucl. Phys. B356 (1991) 154.

54. C. P. Martin, in preparation.

55. A.M. Polyakov, Nucl. Phys. B164 (1979) 171. 
J.L. Gervais and A. Neveu, Nucl. Phys. B163 (1980) 189.

V.S. Dotsenko and S.N. Vergeles, Nucl. Phys. B169 (1980) 527.

56. A. Jaffe and A. Lesniewski, Supersymmetric quantum fields and infinite dimensional analysis, in Non-perturbative quantum field theory, G. 't Hooft, A. Jaffe, G. Mack P.K. Mitter and R. Stora eds., Plenum Press (1988)

57. I. S. Gradshteyn and I. M. Ryzhik, Table of integrals, series and products, XX edition, Academic Press (1980). 


\section{Figures' captions}

Fig. 1: Feynaman rules for the action (2.4).

Fig. 2: One-loop diagrams contributing to the vacuum polarization tensor.

Figs. 3, 4, 5 and 6: Two-loop diagrams contributing to the vacuum polarization tensor.

Fig. 7: One-loop diagram contributing to the ghost self-energy.

Fig. 8: Two-loop diagrams contributing to the ghost self-energy.

Fig. 9: One-loop diagrams contributing to the ghost-ghost-external field vertex.

Fig. 10: Two-loop diagrams contributing to the ghost-ghost-external field vertex. 